Review

\title{
Fluency Interventions for Elementary Students with Reading Difficulties: A Synthesis of Research from 2000-2019
}

\author{
Alida Hudson *(D), Poh Wee Koh, Karol A. Moore and Emily Binks-Cantrell \\ Department of Teaching, Learning, and Culture, Texas A \& M University, 4232 TAMU, College Station, TX 77843, \\ USA; pohweekoh@tamu.edu (P.W.K.); kmoore7495@tamu.edu (K.A.M.); aggieemily@tamu.edu (E.B.-C.) \\ * Correspondence: alida.hudson@tamu.edu
}

Received: 15 January 2020; Accepted: 26 February 2020; Published: 28 February 2020

\begin{abstract}
Oral reading fluency (ORF) deficits are a hallmark of reading difficulties. The impact of fluency struggles extends beyond word-level difficulties to include deficits in reading comprehension. Sixteen empirical studies conducted in 2000-2019 that examined ORF interventions among elementary students identified as having reading difficulties were reviewed to identify the characteristics (e.g., instructional variables, group size, type of interventionist) of effective ORF interventions and their impact on English oral reading fluency and reading comprehension outcomes. The systematic review revealed that interventions reported centered around repeated reading procedures (86.5\%). Across the 16 studies, outcomes for oral reading fluency varied widely and most focused on speed and rate aspects rather than prosody. Effect sizes for rate and accuracy measures ranged from negligible to large (i.e., 0.01 to 1.18) and three studies found large effects for prosody outcomes. Effect sizes for reading comprehension ranged between non-significant and large significant effects. Findings support the use of repeated reading of text to build up ORF of students with reading difficulties. Interventions that were found to be most effective were those that were conducted one-on-one with a trained model of fluent word reading and accuracy. Findings also point to three gaps in our understanding: (1) the efficacy of interventions other than repeated reading, (2) effects of ORF interventions on prosody outcomes, and (3) sustainability of outcomes.
\end{abstract}

Keywords: interventions; oral reading fluency; reading comprehension; reading difficulties; systematic review

\section{Introduction}

According to the most recent scores from the National Assessment of Educational Progress (NAEP), a significant number of fourth- and eighth-grade students in the United States (U.S.) are failing to read at satisfactory levels [1]. Specifically, $32 \%$ of all fourth-grade students were found to be reading below basic levels, demonstrating difficulties with making simple inferences and finding relevant information to support their understanding of a text. This statistic sees an alarming increase among students identified as having learning disabilities, with $68 \%$ of struggling fourth graders reading below basic levels. This is hardly surprising considering that difficulties with reading are commonplace among students identified with learning disabilities [2].

Chall's stages of reading highlight the importance of oral reading fluency as students' decoding develops. Proficient oral reading fluency is one of the main characteristics identifying children moving from stage 2 (learning to read) where the focus is on rapid decoding of words to stage 3 (reading to learn) where students begin to gain new knowledge and ideas through their reading of increasingly complex texts from a variety of different genres [3]. Based on Chall's model, fourth graders should 
be entering the reading-to-learn phase (stage 3). However, findings of students reading below basic levels in the fourth grade on the NAEP suggest that many fourth graders in the U.S. have not entered this phase and are still working to master learning to read. Students' difficulties with oral reading fluency may thus be one factor prohibiting students from progressing to the more advanced stages of reading development [3]. This theoretical proposition is well supported in research conducted among students in the U.S. For instance, Daane, Campbell, Grigg, Goodman, and Oranje examined a subsample of fourth-grade students who took the NAEP reading assessment in 2002 and identified $40 \%$ of the sample to be "non-fluent" readers who also scored at or below the basic achievement level on the overall NAEP reading assessment [4]. The negative impact of deficits in oral reading fluency extends to many other school subjects that require students to gain understanding through reading [5], further underscoring the importance of oral reading fluency for reading development. Beyond the U.S. context, the importance of fluency for reading is also evident in that reading curricula standards across different countries and languages cite the achievement of fluency in reading words and texts, typically by grade three, as a curricula goal [6]. Deficits in automaticity in reading have also been found to be associated with children identified as having reading disabilities across a range of languages $[7,8]$.

Therefore, in light of the far-reaching effects of difficulties in reading fluency and that fluency is identified as a crucial building block of reading development [5,9], it is essential that fluency interventions be provided for elementary students demonstrating difficulties in reading [5]. An increase in oral reading fluency skills would allow students to read more complex text at more proficient levels. Thus, this synthesis of research will examine the types of interventions which aim to build the oral reading fluency and their impact on reading outcomes in students with reading difficulties.

\subsection{Oral Reading Fluency}

Fluent reading is often conceptualized as being synonymous with three aspects, namely, the ability to read texts with accuracy, appropriate rate, and prosody [10], with the ultimate aim of extracting meaning in reading [11]. The multifaceted nature of fluency and the association between fluency and reading comprehension is well-placed in theory and empirical research [11-14]. For one, how rate and accuracy components in reading fluency facilitate reading comprehension is outlined in the model of automatic processing [15]. According to this model, all individuals have limited cognitive capacity which makes it necessary for readers to develop automaticity in lower-level word recognition skills such as grapheme-phoneme correspondences and phonemic awareness in order to dedicate adequate cognitive resources to higher-order tasks involving comprehension of texts [15]. This ability to develop automaticity in word recognition processes is what often differentiates fluent from dysfluent readers. Dysfluent readers likely find their cognitive attention consumed primarily by decoding as they have to intently and laboriously attend to letters, sound-symbol correspondences, and word recognition. The inability to achieve automaticity in lower-order processing places large demands on working memory, leaving few resources available to negotiate meaning making in texts. Considering the centrality of working memory in facilitating the storage and retrieval of information in texts during the process of reading for comprehension [16,17], non-automaticity in decoding hinders reading comprehension [18].

It has also been suggested that fluency aspects of accuracy and rate mediates the association between decoding and reading comprehension, a postulation that has been empirically supported. Silverman, Speece, Harring, and Ritchey measured the contributions of aspects of decoding comprising phonological awareness, word and nonword reading as well as fluency (measured by speed and accuracy in reading words, sentences, and texts) in reading comprehension, controlling for linguistic comprehension [19]. They found that when fluency aspects were not included in the model, there was a significant relation between decoding and reading comprehension. However, this relation was no longer apparent once fluency was accounted for. In addition, students who were good decoders but non-fluent readers were weaker in reading comprehension as compared to those with strong decoding and fluency skills [19]. The finding in Silverman et al. that good decoders could be further classified 
as fluent or dysfluent readers also supports that of previous research, suggesting that although there is a strong association between decoding and fluency [20,21], proficiency in the former does not automatically translate into the latter. Rather, explicit instruction that builds up fluency is necessary to facilitate the transition from being able to recognize words accurately to being able to recognize words both accurately and rapidly [19].

The role of prosody in reading comprehension has also been established, although to a lesser extent than accuracy and rate aspects. Prosody, a frequently overlooked component of oral reading fluency [22], is concerned with reading with expression and is associated with the ability to produce appropriate changes in pitch and stress as well as use appropriate phrasing and intonation [14]. Prosodic elements are suggested to be central components of fluency and reading comprehension because reading words in meaningful phrases allows readers to focus on the ideas in the text and students who read without expression are likely not gaining full meaning of texts [9,23]. Empirical research supports this view. For instance, in a study examining the relationship between prosody and reading comprehension in 80 third-grade students [24], students read aloud a passage which targeted specific characteristics of prosodic reading, including (1) basic declarative sentences thought to elicit a decline in pitch, (2) basic quotes intended to elicit pauses in phrasing, (3) yes/no questions designed to elicit an increase in pitch, and (4) phrase-final commas thought to elicit pauses in phrasing. Findings showed that children who demonstrated greater prosodic awareness, where they showed a decline in pitch in declarative sentences and an ascent in pitch with yes/no questions, had higher reading comprehension scores as those who did not.

\subsection{Oral Reading Fluency and Reading Difficulties}

Increasingly, deficits in reading fluency have been acknowledged as an area in which children with reading disabilities struggle $[20,25]$. This is evident in that oral reading fluency was added to the federal definition of a specific learning disability through the Individuals with Disabilities Education Improvement Act (IDEA) in 2004 [26]. Scientific studies of reading also support this view. For instance, as evidenced in Daane et al. [4], many students with reading difficulties were dysfluent readers who read slowly and laboriously. These students often struggled with automatic word recognition and had to stop frequently while reading to sound out words or use structural analysis to look for common syllable types or morphemes [27]. In another study, Rasinski and Padak examined the word recognition, oral reading fluency, and reading comprehension abilities of 604 students in second through sixth grade identified as having reading difficulties [28]. After collecting data from an informal reading assessment (Ekwall Reading Inventory) and routine assessments given by teachers, Rasinski and Padak concluded that difficulties with reading speed translated into fluency deficits that negatively impacted reading comprehension [28]. In another recent study, Kang and Shin concluded that an estimated $6 \%$ of struggling readers in upper elementary grades exhibited early reading difficulties related to decoding and fluency [29]. Moreover, when combined with students who had issues with basic reading skills, as well as comprehension, more than half of all struggling readers in upper elementary grades displayed deficits in decoding and fluency skills. Their work emphasized that fluency is, in fact, an essential component of reading comprehension, and that fluency, while it overlaps with decoding, is a separate component that makes unique contributions to reading comprehension.

In light of the evidence suggesting the crucial role fluency plays in reading, especially among children with reading disabilities, researchers have pointed to the importance of interventions targeted at raising students' fluency skills. Specifically, researchers have emphasized the need for interventions to target fluency aspects directly rather than indirectly through other related reading skills such as phonological awareness because interventions of the latter nature alone have not been shown to improve fluency skills [5,12,30]. Researchers have put forth that fluency improvements increase reading comprehension among struggling readers indirectly by freeing up limited working memory resources to be used in higher-order processes in reading [15,31,32]. This explanation is tenable considering 
that research has shown that children with reading difficulties often exhibit working memory deficits, see [33] for a review.

However, fluency instruction is often absent from elementary school reading curriculum today, possibly because classroom teachers are rarely provided with any training on instructional strategies to promote oral reading fluency [34]. This lack of attention to fluency in instruction and the consideration that fluency is a skill that has been identified to be difficult to build up points to the importance of examining the efficacy of direct interventions of fluency $[35,36]$. In addition, considering the close association between fluency and reading comprehension, examining the efficacy of fluency interventions on reading comprehension in addition to fluency outcomes is necessary. In general, previous syntheses and meta-analyses that have examined children with reading disabilities have shown oral reading fluency interventions to be effective in improving fluency and/or comprehension outcomes in school-aged children [37-39]. Across these reviews, researchers have also highlighted the importance of considering characteristics including type of intervention, type of interventionist (adult vs. peer), number of times texts were read in the interventions, pre-instructional support, corrective feedback, and number of elements included in interventions in order to assess intervention effectiveness [37-39], although these have not been systematically examined among children with reading difficulties across reviews. For instance, the systematic review conducted by Therrien found considerable effect sizes associated with fluency interventions on fluency and comprehension outcomes for students with and without learning disabilities (LD) and highlighted that interventions that were conducted by an adult typically yielded greater effect sizes as compared to those mediated by peers [39]. However, the review focused solely on repeated reading interventions and did not consider the effects of other types of interventions. In addition, the characteristics of interventions and their effectiveness on reading outcomes for LD children specifically were not discussed. Similarly, only repeated reading interventions were examined in a more recent meta-analysis conducted by Lee and Yoon and the effects of interventions on fluency outcomes only were examined. Transfer effects of fluency to reading comprehension outcomes were not examined [37]. Furthermore, although the review conducted by Chard et al. discussed in detail intervention characteristics related to different fluency interventions and the effects of interventions on both fluency and comprehension outcomes, effect sizes across studies reviewed were not reported [38]. In addition, the number of studies reviewed pertaining to the different intervention characteristics were very small, making it difficult to draw definite conclusions from these studies. Finally, in the most recent synthesis by Stevens et al. [40], single-case studies figured as the majority which the authors noted could impose limits on the generalizability of findings.

\subsection{The Current Study}

In light of these gaps, this paper seeks to systematically examine the effects of oral reading fluency interventions on elementary students (i.e., first through fifth grade) identified as having reading difficulties across a range of experimental studies conducted in school settings. Specifically, we addressed the following objectives: (1) whether oral reading fluency interventions increase the oral reading fluency (i.e., accuracy, rate, and prosody) and reading comprehension of elementary students identified as having reading difficulties, and (2) the characteristics of effective interventions that facilitate these reading outcomes.

In addition to providing a more updated examination of the effectiveness of fluency interventions, the current review builds on previous syntheses [37-40] in several ways. First, we expanded our examination of types of fluency interventions to include that other than repeated reading. Second, we examined the magnitude of intervention effects on both fluency and comprehension outcomes within the same synthesis to examine the local and transfer effects of interventions, respectively, and compared the magnitude of effects of interventions on both types of outcomes. Third, our present study only reviewed group-design studies, following Stevens et al.'s point about limitations on generalization of findings with single-case designs [40]. Furthermore, we only included studies that had a control group in order to control for quality of study design and to allow for more definite conclusions to be 
made about the effectiveness of fluency interventions. Finally, although previous syntheses did not examine whether group size influenced the effectiveness of interventions, we consider this variable in our present study. This is because intervention studies pertaining to different reading constructs have yielded different findings regarding optimal group size for interventions [41,42]. Thus, it is necessary to examine whether this variable influences intervention outcomes.

\section{Methods}

\subsection{Selection Procedures}

Several databases [i.e., PsycINFO, Education Source, Academic Search Ultimate and Education Resources Information Center (ERIC)] were searched using the terms oral reading fluency, and intervention*, repeated reading, choral reading, partner reading, assisted reading, readers' theater, and reading difficulty ${ }^{*}$, struggling reader ${ }^{*}$, or reading disability* and elementary, primary, or K-5. Since the National Reading Panel (NRP) report identified fluency as an essential component of reading instruction which led to increased interest in fluency research $[5,43,44]$, only peer-reviewed papers published after the NRP report (i.e., 2000-2019) that discussed fluency interventions' impact on elementary students with reading difficulties or learning disabilities were identified to be reviewed. Selecting papers that have undergone the peer-review process, which carefully inspects studies for publication, helps to ensure the findings align with the most recent evidence-based practices in fluency instruction. In addition, the references cited in recent meta-analysis and synthesis papers examining oral reading fluency interventions were inspected for applicable papers to review $[37,45]$. Finally, each article's cited references were searched for relevant papers.

The initial search resulted in 365 studies (see Figure 1). Thirty-two articles were duplicates and therefore immediately removed. The titles, keywords, and abstracts of the remaining 333 studies were reviewed for exclusion criteria. Studies were excluded if the title, keywords, or abstracts clearly identified that the study (1) was a synthesis, meta-analysis, or review paper, (2) included students not enrolled in kindergarten through fifth grade, (3) contained instruction in languages other than English, (4) included students with cognitive, visual, or hearing deficits, or (5) did not target oral reading fluency intervention. Using this exclusion criteria, 199 studies were excluded based on title, keyword, or abstracts, leaving 134 studies for further review. The following inclusion criteria were used for an in-depth analysis of the 134 studies.

1. Articles had participants who were described as at-risk for or having reading difficulties. Articles with learning difficulties, struggling readers, at-risk readers, below level readers, and learning-disabled readers were all included. Articles were included if they provided segregated data for students with reading difficulties if students with reading difficulties were part of a larger group of participants. Articles with average or above-average readers were excluded.

2. Studies used experimental or quasi-experimental designs with a treatment and control group. Studies using single-case designs were excluded. Studies which did not include a no-treatment control group were excluded. Meta-analysis, commentaries, or other synthesis papers were excluded.

3. Participants were elementary-age students (i.e., kindergarten through fifth grade). Articles were included if they provided segregated data for elementary students if students were part of a larger group of participants. Articles with less than $50 \%$ of participants enrolled in elementary school were excluded.

4. Fluency intervention was delivered in English in a school setting. Studies with the intervention being delivered at home or in a clinic setting were excluded. Studies in languages other than English were excluded.

5. Articles focused on the implementation of an oral reading fluency intervention that aimed to improve students' oral reading fluency. Articles were included if at least $50 \%$ of a multi-component 
intervention was targeting oral reading fluency. Studies not including oral reading fluency of connected text as a dependent measure were excluded.

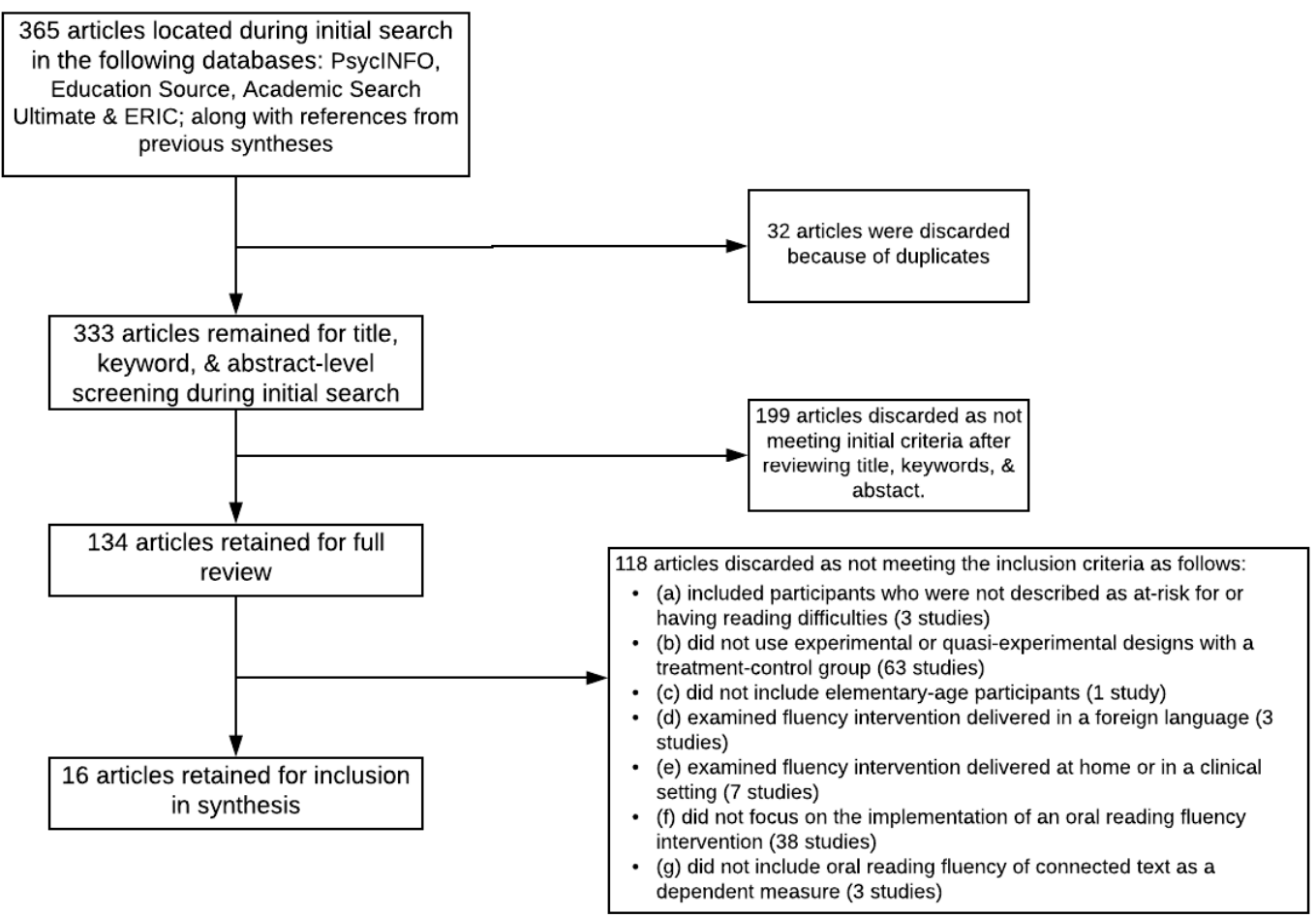

Figure 1. The Preferred Reporting items for Systematic Reviews and Meta-Analyses Flowchart.

After review, 16 of the 134 studies comprehensively reviewed met the criteria for inclusion; 118 studies were excluded because they (a) included participants who were not described as at risk for or having reading difficulties (3 studies), (b) did not use experimental or quasi-experimental designs with a treatment-control group (63 studies), (c) did not include elementary-age participants (1 study), (d) examined fluency intervention delivered in a foreign language (3 studies), (e) examined fluency intervention delivered at home or in a clinical setting (7 studies), (f) did not focus on the implementation of an oral reading fluency intervention (38 studies), or (g) did not include oral reading fluency of connected text as a dependent measure (3 studies).

\subsection{Coding Procedures}

The final 16 studies underwent an inclusive coding protocol by the first author. Twenty percent of the studies were double coded by the first and third authors; $94 \%$ interrater agreement was achieved, and discrepancies in coding were resolved via discussion. The following categories were included in all coding procedures: (a) author and study design; (b) participant information, including if English Language Learners were included in the participants; (c) treatment description, including group size, duration, and other intervention variables such as text level used and number of reads; (e) ORF dependent measures; (f) reading comprehension dependent measures if applicable; and (f) findings. See Table 1 for study information. 
Table 1. Study Information.

\begin{tabular}{|c|c|c|c|c|c|c|c|c|}
\hline Article Info & $\begin{array}{l}\text { Study } \\
\text { Design }\end{array}$ & $\begin{array}{l}\text { Participant } \\
\text { Information }\end{array}$ & Intervention & $\begin{array}{c}\text { Multiple } \\
\text { Features of } \\
\text { Intervention }\end{array}$ & Intervention Description & Group Size & $\begin{array}{l}\text { Intervention } \\
\text { Duration }\end{array}$ & $\begin{array}{c}\text { Outcomes } \\
\text { Effect Sizes } \\
\text { Calculated Using } \\
\text { Carlson and } \\
\text { Schmidt, 1999 }\end{array}$ \\
\hline [46] & $\mathrm{E}$ & $\begin{array}{c}100 \text { second-grade } \\
\text { students } \\
\text { (50 Treatment, } 50 \\
\text { Control) } \\
\text { 11\% White, } 57 \% \text { African } \\
\text { American, } 24 \% \\
\text { Hispanic, } 4 \% \text { Asian, } 4 \% \\
\text { other } \\
52 \text { Males }\end{array}$ & $\begin{array}{l}\text { Repeated } \\
\text { Reading with } \\
\text { Multiple } \\
\text { Features }\end{array}$ & $\begin{array}{l}\text { ChR } \\
\text { MFR } \\
\text { EC }\end{array}$ & $\begin{array}{l}\text { Independent level text }(95 \% \\
\text { accuracy or above). } \\
3 \text { reads: } \\
\text { (1) choral reading of text (peers } \\
\text { read aloud together at same } \\
\text { pace); } \\
\text { (2) alternate reading of text } \\
\text { sentence by sentence; } \\
\text { (3) weaker student reads text } \\
\text { with stronger student helping } \\
\text { with unknown words; } \\
\text { (4) 1-minute timed reading and } \\
\text { chart progress. }\end{array}$ & $\begin{array}{l}\text { 1:1 with Peer } \\
\text { Coach }\end{array}$ & $\begin{array}{c}36 \text { weeks } \\
10-12 \text { min. } \\
3 \text { times/week }\end{array}$ & $\begin{array}{c}\text { ORF: } \\
\text { DORF: RRMF > CG } \\
(\mathrm{es}=1.06)\end{array}$ \\
\hline [47] & QE & $\begin{array}{c}59 \text { second-grade } \\
\text { students } \\
\text { (29 treatment, } 30 \\
\text { control) } \\
52.5 \% \text { White, } 28.8 \% \\
\text { African American, } \\
\text { 15.3\% Latino } \\
\text { 27 Males }\end{array}$ & $\begin{array}{l}\text { Repeated } \\
\text { Reading with } \\
\text { Multiple } \\
\text { Features }\end{array}$ & $\begin{array}{l}\text { MFR } \\
\text { PD } \\
\text { PF } \\
\text { VC } \\
\text { GS }\end{array}$ & $\begin{array}{l}\text { Delivered by a teacher. } \\
\text { 3-4 reads. } \\
\text { Begins with verbal cue (a } \\
\text { reminder such as "Read this } \\
\text { story the best you can and as } \\
\text { quickly as you can."). } \\
\text { Teacher times student reading } \\
\text { and student provides retell. If } \\
\text { goal is met, new passage is } \\
\text { given. If goal is not met, } \\
\text { student continues to work with } \\
\text { the same passage. } \\
\text { After final read, graph WCPM } \\
\text { progress and praise student. }\end{array}$ & $1: 1$ & $\begin{array}{c}20 \text { weeks } \\
10 \text { min. } \\
\text { 2-3 times/week }\end{array}$ & $\begin{array}{c}\text { ORF: } \\
\text { DORF: RRMF > CG } \\
\text { (es }=1.18) \\
\text { GORT-F: RRMF > } \\
\text { CG (es }=0.56) \\
\text { RC: } \\
\text { GORT-C: RRMF > } \\
\text { CG }(\text { es }=0.70)\end{array}$ \\
\hline
\end{tabular}


Table 1. Cont

\begin{tabular}{|c|c|c|c|c|c|c|c|c|}
\hline Article Info & $\begin{array}{l}\text { Study } \\
\text { Design }\end{array}$ & $\begin{array}{l}\text { Participant } \\
\text { Information }\end{array}$ & Intervention & $\begin{array}{l}\text { Multiple } \\
\text { Features of } \\
\text { Intervention }\end{array}$ & Intervention Description & Group Size & $\begin{array}{l}\text { Intervention } \\
\text { Duration }\end{array}$ & $\begin{array}{c}\text { Outcomes } \\
\text { Effect Sizes } \\
\text { Calculated Using } \\
\text { Carlson and } \\
\text { Schmidt, } 1999\end{array}$ \\
\hline [48] & E & $\begin{array}{c}21 \text { second-grade } \\
\text { students } \\
\text { (13 Treatment, } 8 \\
\text { Control) } \\
\text { 95.2\% Latino } \\
16 \text { Males }\end{array}$ & $\begin{array}{l}\text { Repeated } \\
\text { Reading with } \\
\text { Multiple } \\
\text { Features }\end{array}$ & $\begin{array}{l}\text { MFR } \\
\text { PD } \\
\text { PF } \\
\text { VC } \\
\text { GS }\end{array}$ & $\begin{array}{l}\text { Delivered by a teacher. } \\
3-4 \text { reads. } \\
\text { Begins with verbal cue (a } \\
\text { reminder such as "Read this } \\
\text { story the best you can and as } \\
\text { quickly as you can."). } \\
\text { Teacher times student reading } \\
\text { and student provides retell. If } \\
\text { goal is met, new passage is } \\
\text { given. If goal is not met, } \\
\text { student continues to work with } \\
\text { the same passage. } \\
\text { After final read, graph WCPM } \\
\text { progress and praise. }\end{array}$ & $1: 1$ & $\begin{array}{c}20 \text { weeks } \\
10 \text { min. } \\
2-3 \text { times/week }\end{array}$ & $\begin{array}{c}\text { ORF: } \\
\text { GORT-F: RRMF > } \\
\text { CG (es = 0.95) } \\
\text { RC: } \\
\text { GORT-C: RRMF > } \\
\text { CG (es }=1.12)\end{array}$ \\
\hline [49] & QE & $\begin{array}{c}20 \text { upper elementary } \\
\text { students } \\
\text { (10 Treatment, } 10 \\
\text { Control) }\end{array}$ & $\begin{array}{l}\text { Listening While } \\
\text { Reading }\end{array}$ & Audiobooks & $\begin{array}{l}\text { Choice of text at or below } \\
\text { reading level. } \\
\text { Each intervention student had } \\
\text { an MP3 player with an } \\
\text { audiobook downloaded on the } \\
\text { device as well as a hard copy of } \\
\text { the book that corresponded the } \\
\text { audiobook. New audiobooks } \\
\text { and physical books were } \\
\text { provided to the student as } \\
\text { needed. } \\
\text { Control group students } \\
\text { participated in independent } \\
\text { silent reading. }\end{array}$ & $1: 1$ & $\begin{array}{c}8 \text { weeks } \\
20-30 \text { min. } \\
4-5 \text { times/week }\end{array}$ & $\begin{array}{c}\text { ORF: } \\
\text { DORF: } \text { LWR > CG } \\
(\mathrm{es}=0.64)\end{array}$ \\
\hline
\end{tabular}


Table 1. Cont

\begin{tabular}{|c|c|c|c|c|c|c|c|c|}
\hline Article Info & $\begin{array}{l}\text { Study } \\
\text { Design }\end{array}$ & $\begin{array}{l}\text { Participant } \\
\text { Information }\end{array}$ & Intervention & $\begin{array}{c}\text { Multiple } \\
\text { Features of } \\
\text { Intervention }\end{array}$ & Intervention Description & Group Size & $\begin{array}{l}\text { Intervention } \\
\text { Duration }\end{array}$ & $\begin{array}{l}\text { Outcomes } \\
\text { Effect Sizes } \\
\text { Calculated Using } \\
\text { Carlson and } \\
\text { Schmidt, } 1999\end{array}$ \\
\hline [50] & E & $\begin{array}{l}46 \text { third-grade students } \\
\text { (23 Treatment, } 23 \\
\text { Control) } \\
12 \text { Males }\end{array}$ & $\begin{array}{l}\text { Listening While } \\
\text { Reading }\end{array}$ & Audiobooks & $\begin{array}{c}\text { Each intervention student had } \\
\text { a tablet loaded with } 60 \\
\text { children's audiobooks. The } \\
\text { students listened and read } \\
\text { along to one audiobook each } \\
\text { day. } \\
\text { Students in the no intervention } \\
\text { control group read a book but } \\
\text { were not provided with the } \\
\text { audiobook to accompany the } \\
\text { text. }\end{array}$ & $1: 1$ & $\begin{array}{l}4 \text { weeks } \\
10 \text { min. } \\
\text { Daily }\end{array}$ & $\begin{array}{c}\text { ORF: } \\
\text { Experimental: LWR } \\
>\text { CG }(\mathrm{es}=0.07)\end{array}$ \\
\hline [51] & E & $\begin{array}{c}24 \text { second-grade } \\
\text { students } \\
\text { (6 RRMF, 6 Continuous } \\
\text { Reading, 6 Listening } \\
\text { only, 6 Control) } \\
\text { 79\% African American, } \\
\text { 17\% White, } 4 \% \\
\text { Hispanic } \\
10 \text { Males }\end{array}$ & $\begin{array}{l}\text { (1) Repeated } \\
\text { Reading with } \\
\text { Multiple } \\
\text { Features } \\
\text { (2) Continuous } \\
\text { Reading } \\
\text { (3) Listening } \\
\text { Only }\end{array}$ & $\begin{array}{l}\text { (1) ER, ChR, } \\
\text { Positive } \\
\text { Feedback, Oral } \\
\text { Rendition of } \\
\text { Practiced Texts } \\
\text { (2) ER, ChR }\end{array}$ & $\begin{array}{l}\text { Repeated Reading: Read one } \\
\text { story } 3 \text { to } 4 \text { times over the } \\
\text { course of the three sessions. } \\
\text { Day 1: Echo and Choral } \\
\text { Reading (students mimic } \\
\text { teacher's reading or teacher } \\
\text { read aloud same text together, } \\
\text { respectively Day 2: Partner } \\
\text { Reading (students read } \\
\text { alternate pages); Day 3: Choral } \\
\text { Reading and Oral Performance } \\
\text { for Small Group. } \\
\text { Continuous Reading: A single } \\
\text { reading of a different story at } \\
\text { each session. Echo and Choral } \\
\text { Reading of text (students } \\
\text { mimic teacher's reading or } \\
\text { teacher read aloud same text } \\
\text { together, respectively). } \\
\text { Listening Only: Adult reads } \\
\text { story aloud with expression. }\end{array}$ & Small Group & $\begin{array}{c}6 \text { weeks } \\
15-20 \mathrm{~min} \\
3 \text { times/week }\end{array}$ & 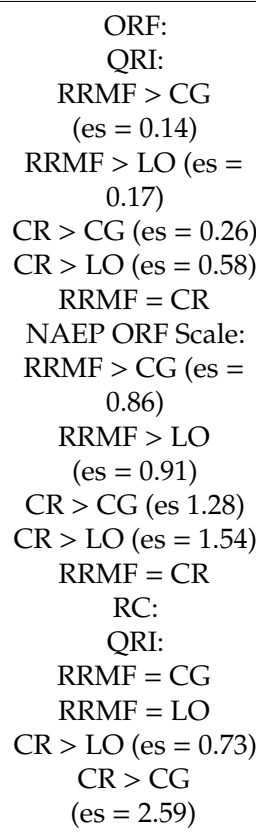 \\
\hline
\end{tabular}


Table 1. Cont

\begin{tabular}{|c|c|c|c|c|c|c|c|c|}
\hline Article Info & $\begin{array}{l}\text { Study } \\
\text { Design }\end{array}$ & $\begin{array}{l}\text { Participant } \\
\text { Information }\end{array}$ & Intervention & $\begin{array}{c}\text { Multiple } \\
\text { Features of } \\
\text { Intervention }\end{array}$ & Intervention Description & Group Size & $\begin{array}{l}\text { Intervention } \\
\text { Duration }\end{array}$ & $\begin{array}{c}\text { Outcomes } \\
\text { Effect Sizes } \\
\text { Calculated Using } \\
\text { Carlson and } \\
\text { Schmidt, } 1999\end{array}$ \\
\hline [52] & E & $\begin{array}{c}34 \text { second-grade } \\
\text { students } \\
\text { (17 Treatment, } 17 \\
\text { Control) } \\
57 \% \text { African American, } \\
24 \% \text { Hispanic, } 11 \% \\
\text { White, } 4 \% \text { Asian, } 4 \% \\
\text { other } \\
18 \text { Males }\end{array}$ & $\begin{array}{c}\text { Repeated } \\
\text { Reading with } \\
\text { Multiple } \\
\text { Features }\end{array}$ & $\begin{array}{l}\text { ChR } \\
\text { MFR } \\
\text { EC }\end{array}$ & $\begin{array}{l}\text { Independent level text ( } 95 \% \\
\text { accuracy or above). } \\
3 \text { reads: } \\
\text { (1) choral reading of text (peers } \\
\text { read aloud together at same } \\
\text { pace); } \\
\text { (2) alternate reading of text } \\
\text { sentence by sentence; } \\
\text { (3) weaker student reads text } \\
\text { with stronger student helping } \\
\text { with unknown words. }\end{array}$ & $\begin{array}{l}\text { 1:1 with Peer } \\
\text { Coach }\end{array}$ & $\begin{array}{c}36 \text { weeks } \\
10-12 \text { min. } \\
3 \text { times/week }\end{array}$ & $\begin{array}{c}\text { ORF: } \\
\text { DORF: RRMF > CG } \\
\text { (es = 1.12) }\end{array}$ \\
\hline [53] & E & $\begin{array}{c}30 \text { students } \\
10 \text { 2nd grade, } 20 \text { 3rd } \\
\text { grade } \\
97 \% \text { African American, } \\
\text { 3\% Hispanic } \\
10 \text { Males }\end{array}$ & $\begin{array}{l}\text { Repeated } \\
\text { Reading with } \\
\text { Multiple } \\
\text { Features }\end{array}$ & $\begin{array}{l}\text { PD } \\
\text { LPP }\end{array}$ & $\begin{array}{l}\text { After school program. } \\
\text { Instructional level text } \\
\text { ( } 90 \%-94 \% \text { accuracy). } \\
\text { The adult and student } \\
\text { alternated reading the story } 2 \\
\text { times each. } \\
\text { If } 100 \text { WCPM was reached on a } \\
\text { previously read text after a } \\
\text { two-day retention period, a } \\
\text { more difficult text was used. }\end{array}$ & $1: 1$ & $\begin{array}{l}7 \text { weeks (2nd } \\
\text { grade), } 8 \text { weeks } \\
\text { (3rd grade) } \\
30 \text { min. } \\
3 \text { times/week }\end{array}$ & $\begin{array}{c}\text { ORF: } \\
\text { CBM-R probes: } \\
\text { Immediate: RRMF } \\
\text { > CG; Retention: } \\
\text { RRMF > CG } \\
\text { 2nd Grade: } \\
\text { CBM grade 2: es = } \\
0.14 \\
\text { CBM grade } 3 \text { es = } \\
-0.06 \\
\text { CBM grade } 4 \text { es = } \\
-0.24 \\
\text { 3rd Grade: } \\
\text { CBM grade 2: es = } \\
0.13 \\
\text { CBM grade } 3 \text { es = } \\
-0.06 \\
\text { CBM grade } 4 \text { es = } \\
0.05\end{array}$ \\
\hline
\end{tabular}


Table 1. Cont

\begin{tabular}{|c|c|c|c|c|c|c|c|c|}
\hline Article Info & $\begin{array}{l}\text { Study } \\
\text { Design }\end{array}$ & $\begin{array}{l}\text { Participant } \\
\text { Information }\end{array}$ & Intervention & $\begin{array}{c}\text { Multiple } \\
\text { Features of } \\
\text { Intervention }\end{array}$ & Intervention Description & Group Size & $\begin{array}{l}\text { Intervention } \\
\text { Duration }\end{array}$ & $\begin{array}{c}\text { Outcomes } \\
\text { Effect Sizes } \\
\text { Calculated Using } \\
\text { Carlson and } \\
\text { Schmidt, } 1999\end{array}$ \\
\hline [54] & $\mathrm{E}$ & $\begin{array}{c}37 \text { students } \\
\text { 16 2nd grade, } 214 \text { th } \\
\text { grade } \\
\text { (21 Treatment, 16 } \\
\text { Control) } \\
50 \% \text { White, } 29 \% \\
\text { Hispanic, 18\% African } \\
\text { American, 3\% Other }\end{array}$ & $\begin{array}{l}\text { (1) Repeated } \\
\text { Reading with } \\
\text { Multiple } \\
\text { Features } \\
\text { (2) Continuous } \\
\text { Reading }\end{array}$ & $\begin{array}{l}\text { (1) EC } \\
\text { (2) EC }\end{array}$ & $\begin{array}{l}\text { Instructional level text used for both } \\
\text { conditions ( } 90 \%-94 \% \text { accuracy). } \\
\text { Repeated Reading+EC: Read each } \\
\text { page of a text } 3 \text { times, teacher } \\
\text { provided error correction as needed. } \\
\text { Continuous Reading: Read from } \\
\text { same book without repeating pages, } \\
\text { teacher provided error correction as } \\
\text { needed. }\end{array}$ & $1: 1$ & $\begin{array}{c}14 \text { weeks } \\
15 \text { min. } \\
3 \text { times/week }\end{array}$ & $\begin{array}{c}\text { ORF: } \\
\text { GORT4: RRMF > } \\
\text { CG (es =0.53); CR > } \\
\text { CG (es = 0.58); } \\
\text { RRMF = CR } \\
\text { RC: } \\
\text { WRMT-PC: RRMF > } \\
\text { CG (es = 1.09); CR > } \\
\text { CG (es = 0.71); } \\
\text { GORT-C: } \\
\text { RRMF > CG (es = } \\
0.75) ; \\
\text { CR > CG (es = 0.95) } \\
\text { RRMF = CR }\end{array}$ \\
\hline [55] & $\mathrm{QE}$ & $\begin{array}{c}119 \text { students } \\
\text { 3rd- 6th Grade } \\
\text { (59 Treatment, } 60 \\
\text { Control) }\end{array}$ & $\begin{array}{l}\text { Repeated } \\
\text { Reading with } \\
\text { Multiple } \\
\text { Features }\end{array}$ & $\begin{array}{c}\text { MFR } \\
\text { EC } \\
\text { Summarizing } \\
\text { Predicting }\end{array}$ & $\begin{array}{l}3 \text { steps to each session: } \\
\text { Partner Reading: Stronger reader } \\
\text { read for } 5 \text { min and then weaker } \\
\text { reader read for } 5 \text { min with stronger } \\
\text { reader providing error correction as } \\
\text { needed. Weaker reader retold story } \\
\text { after reading. } \\
\text { Summarizing: Stronger reader read } \\
\text { for } 5 \text { min and then weaker reader } \\
\text { read for } 5 \text { min with stronger reader } \\
\text { providing error correction as needed. } \\
\text { Student reading stopped after each } \\
\text { paragraph to summarize what was } \\
\text { read. } \\
\text { Predicting: Stronger reader read for } \\
5 \text { min and then weaker reader read } \\
\text { for } 5 \text { min with stronger reader } \\
\text { providing error correction as needed. } \\
\text { Student made prediction before } \\
\text { reading, read to check prediction, } \\
\text { and provided summary. }\end{array}$ & $\begin{array}{l}\text { 1:1 with Peer } \\
\text { Coach }\end{array}$ & $\begin{array}{c}15 \text { weeks } \\
35 \text { min. } \\
3 \text { times/week }\end{array}$ & $\begin{array}{c}\text { ORF: } \\
\text { CRAB: ELL+LD es= } \\
\text { 0.33; ELL+LA es = } \\
0.01 \\
\text { RC: } \\
\text { CRAB: ELL+LD es } \\
=1.15 ; \text { ELL+LA es } \\
=0.83\end{array}$ \\
\hline
\end{tabular}


Table 1. Cont

\begin{tabular}{|c|c|c|c|c|c|c|c|c|}
\hline Article Info & $\begin{array}{c}\text { Study } \\
\text { Design }\end{array}$ & $\begin{array}{c}\text { Participant } \\
\text { Information }\end{array}$ & Intervention & $\begin{array}{c}\text { Multiple } \\
\text { Features of } \\
\text { Intervention }\end{array}$ & Intervention Description & Group Size & $\begin{array}{l}\text { Intervention } \\
\text { Duration }\end{array}$ & $\begin{array}{c}\text { Outcomes } \\
\text { Effect Sizes } \\
\text { Calculated Using } \\
\text { Carlson and } \\
\text { Schmidt, } 1999\end{array}$ \\
\hline [56] & $\mathrm{QE}$ & $\begin{array}{c}30 \text { students } \\
134 \text { th grade, } 105 \text { th } \\
\text { grade, } 67 \text { th grade, and } \\
18 \text { th grade } \\
\text { (16 Treatment, } 14 \\
\text { Control) } \\
16 \text { Males }\end{array}$ & $\begin{array}{l}\text { Repeated } \\
\text { Reading with } \\
\text { Multiple } \\
\text { Features }\end{array}$ & $\begin{array}{l}\mathrm{VC} \\
\mathrm{EC} \\
\mathrm{QA}\end{array}$ & $\begin{array}{l}\text { Instructional level text adjusted } \\
\text { based on prior performance } \\
\text { (90\%-94\% accuracy). } \\
\text { Instructional steps: } \\
\text { (1) Verbal Cue (a reminder such as } \\
\text { "Read this story the best you can and } \\
\text { as quickly as you can."); } \\
\text { (2) Question Generation; } \\
\text { (3) Read and Reread story until goal } \\
\text { WCPM reached (2-4 reads); } \\
\text { (4) Error Correction; } \\
\text { (5) Question Answering. }\end{array}$ & $1: 1$ & $\begin{array}{c}16 \text { weeks } \\
10-15 \text { min. }\end{array}$ & $\begin{array}{c}\text { ORF: } \\
\text { DORF: RRMF > CG } \\
\text { (es }=0.38)\end{array}$ \\
\hline [57] & E & $\begin{array}{c}119 \text { students } \\
654 \text { th and } 545 \text { th } \\
\text { (54 Treatment, } 65 \\
\text { Control) } \\
40 \% \text { African American, } \\
24 \% \text { White, } 14 \% \text { Other, } \\
13 \% \text { Hispanic, } 9 \% \\
\text { Asian) } \\
55 \text { Males }\end{array}$ & $\begin{array}{l}\text { Repeated } \\
\text { Reading with } \\
\text { Multiple } \\
\text { Features }\end{array}$ & $\begin{array}{c}\text { MFR } \\
\text { ChR } \\
\text { Vocabulary } \\
\text { Instruction }\end{array}$ & $\begin{array}{c}\text { Delivered by trained } \\
\text { paraprofessional. } \\
\text { Nonfiction passages with } 98 \% \text { of the } \\
\text { words used in the texts being } \\
\text { high-frequency words or words that } \\
\text { reflect grade-level phonics and } \\
\text { syllable patterns } \\
\text { Instructional Steps: } \\
\text { (1) Vocabulary instruction (introduce } \\
\text { new vocabulary prior to reading); } \\
\text { (2) Students take turns reading } \\
\text { passage; } \\
\text { (3) Choral reading of text two times } \\
\text { (students and teacher read aloud } \\
\text { together at the same pace); } \\
\text { (4) } 1 \text { min timed reading; } \\
\text { (5) Question Answering; } \\
\text { (6) Vocabulary instruction (review } \\
\text { vocabulary from reading); } \\
\text { (7) Repeat steps with a 2nd passage. }\end{array}$ & Small Group & $\begin{array}{c}20 \text { weeks } \\
30 \text { min } \\
4 \text { days/week }\end{array}$ & $\begin{array}{c}\text { ORF: } \\
\text { DORF: RRMF = CG } \\
\text { RC: } \\
\text { WRMT-R/NU: } \\
\text { RRMF > CG (es = } \\
0.35)\end{array}$ \\
\hline
\end{tabular}


Table 1. Cont.

\begin{tabular}{|c|c|c|c|c|c|c|c|c|}
\hline $\begin{array}{c}\text { Article } \\
\text { Info }\end{array}$ & $\begin{array}{l}\text { Study } \\
\text { Design }\end{array}$ & $\begin{array}{c}\text { Participant } \\
\text { Information }\end{array}$ & Intervention & $\begin{array}{c}\text { Multiple } \\
\text { Features of } \\
\text { Intervention }\end{array}$ & Intervention Description & Group Size & $\begin{array}{l}\text { Intervention } \\
\text { Duration }\end{array}$ & $\begin{array}{c}\text { Outcomes } \\
\text { Effect Sizes Calculated Using Carlson } \\
\text { and Schmidt, } 1999\end{array}$ \\
\hline [58] & $\mathrm{E}$ & $\begin{array}{c}162 \text { students } \\
110 \text { 2nd grade, } 52 \text { 3rd } \\
\text { grade } \\
\text { (82 Treatment, } 80 \\
\text { Control) } \\
30 \% \text { White, } 28 \% \\
\text { African American, } \\
23 \% \text { Hispanic, } 16 \% \\
\text { Asian, 3\% Other } \\
98 \text { Males }\end{array}$ & $\begin{array}{l}\text { Repeated Reading } \\
\text { with Multiple } \\
\text { Features }\end{array}$ & $\begin{array}{l}\text { MFR } \\
\text { ChR } \\
\text { Phonics/Word-Level } \\
\text { Instruction } \\
\text { EC }\end{array}$ & $\begin{array}{c}\text { Delivered by paraprofessional. } \\
\text { Nonfiction passages with } 98 \% \text { of } \\
\text { the words used in the texts being } \\
\text { high-frequency words or words } \\
\text { that reflect grade-level phonics and } \\
\text { syllable patterns } \\
\text { Instructional Steps: } \\
\text { (1) Phonics instruction } \\
\text { (letter-sound correspondence } \\
\text { practice); } \\
\text { (2) Students take turns reading } \\
\text { passage } \\
\text { (3) Choral reading of text two } \\
\text { times (students and teacher read } \\
\text { aloud together at the same pace). } \\
\text { (4) 1-minute timed reading } \\
\text { (5) Question Answering } \\
\text { (6) Repeat steps with a 2nd } \\
\text { passage. }\end{array}$ & Small Group & $\begin{array}{l}15 \text { weeks } \\
30 \text { min. } \\
4 \text { days/week }\end{array}$ & $\begin{array}{c}\text { ORF: } \\
\text { DORF-Uniform: RRMF }>\text { CG } \\
\text { (es }=0.33) \\
\text { DORF-Alternate: RRMF > CG } \\
\text { (es }=0.46) \\
\text { GORT-4: RRMF }>\text { CG } \\
\text { (es }=0.41) \\
\text { RC: } \\
\text { GORT-4 Comprehension: RRMF = CG }\end{array}$ \\
\hline [59] & $\mathrm{E}$ & $\begin{array}{c}202 \text { students } \\
132 \text { 2nd grade, } 70 \text { 3rd } \\
\text { grade } \\
\text { (98 Treatment, } 104 \\
\text { Control) } \\
33 \% \text { White, } 28 \% \\
\text { Hispanic, } 21 \% \\
\text { African American, } \\
11 \% \text { Asian, } 7 \% \text { Other } \\
119 \text { Males }\end{array}$ & $\begin{array}{l}\text { Repeated Reading } \\
\text { with Multiple } \\
\text { Features }\end{array}$ & $\begin{array}{c}\text { MFR } \\
\text { ChR } \\
\text { Phonics/Word-Level } \\
\text { Instruction } \\
\text { EC }\end{array}$ & $\begin{array}{c}\text { Delivered by classroom teacher or } \\
\text { paraprofessional } \\
\text { Nonfiction passages with } 98 \% \text { of } \\
\text { the words used in the texts being } \\
\text { high-frequency words or words } \\
\text { that reflect grade-level phonics and } \\
\text { syllable patterns } \\
\text { Instructional Steps: } \\
\text { (1) Phonics instruction } \\
\text { (letter-sound correspondence } \\
\text { practice); } \\
\text { (2) Students take turns reading } \\
\text { passage; } \\
\text { (3) Choral reading of text two } \\
\text { times (students and teacher read } \\
\text { aloud together at the same pace); } \\
\text { (4) 1 min timed reading; } \\
\text { (5) Question Answering; } \\
\text { (6) Repeat steps with a 2nd } \\
\text { passage. }\end{array}$ & Small Group & $\begin{array}{l}15 \text { weeks } \\
30 \text { min. } \\
4 \text { days/week }\end{array}$ & $\begin{array}{c}\text { Teacher: } \\
\text { ORF: } \\
\text { DORF-Uniform: RRMF > CG (es = 0.46) } \\
\text { DORF-Alternate: RRMF = CG } \\
\text { GORT-4: RRMF > CG }(\mathrm{es}=0.53) \\
\text { RC: } \\
\text { WRMT-R/NU: RRMF > CG } \\
\text { (es = 0.36) } \\
\text { GORT-4 Comprehension: RRMF > CG } \\
\text { (es = 0.10) } \\
\text { Paraprofessional: } \\
\text { ORF: } \\
\text { DORF-Uniform: RRMF > CG }(\mathrm{es}=0.31) \\
\text { DORF-Alternate: RRMF = CG } \\
\text { GORT-4: RRMF > CG (es = 0.32) } \\
\text { RC: } \\
\text { WRMT-R/NU: RRMF > CG (es = 0.14) } \\
\text { GORT-4 Comprehension: RRMF > CG } \\
\text { (es }=-0.12)\end{array}$ \\
\hline
\end{tabular}


Table 1. Cont.

\begin{tabular}{|c|c|c|c|c|c|c|c|c|}
\hline $\begin{array}{l}\text { Article } \\
\text { Info }\end{array}$ & $\begin{array}{l}\text { Study } \\
\text { Design }\end{array}$ & $\begin{array}{l}\text { Participant } \\
\text { Information }\end{array}$ & Intervention & $\begin{array}{l}\text { Multiple } \\
\text { Features of } \\
\text { Intervention }\end{array}$ & Intervention Description & Group Size & $\begin{array}{c}\text { Intervention } \\
\text { Duration }\end{array}$ & $\begin{array}{c}\text { Outcomes } \\
\text { Effect Sizes Calculated Using } \\
\text { Carlson and Schmidt, } 1999\end{array}$ \\
\hline [60] & QE & $\begin{array}{c}52 \text { students } \\
19 \text { 3rd, } 21 \text { th, 12 5th } \\
\text { (29 Treatment, 23 } \\
\text { Control) } \\
88 \% \text { Hispanic, 12\% } \\
\text { White } \\
27 \text { Males }\end{array}$ & $\begin{array}{l}\text { Repeated Reading } \\
\text { with Multiple } \\
\text { Features }\end{array}$ & $\mathrm{RR}+\mathrm{NIM}$ & $\begin{array}{c}\text { Interventional level text (one } \\
\text { year above student's reading } \\
\text { level). } \\
\text { Instructional Steps: } \\
\text { (1) Teacher and student read a } \\
\text { page or paragraph aloud with } \\
\text { the teacher reading slightly } \\
\text { ahead of the student; } \\
\text { (2) Student rereads the page or } \\
\text { paragraph aloud independently; } \\
\text { (3) Repeat steps until time is } \\
\text { complete }\end{array}$ & $1: 1$ & $\begin{array}{l}4 \text { weeks } \\
20 \text { min. } \\
\text { Daily }\end{array}$ & $\begin{array}{c}\text { ORF: } \\
\text { DORF: RRMF > CG }(\mathrm{es}=0.68) \\
\text { MFS: RRMF > CG }(\mathrm{es}=0.98)\end{array}$ \\
\hline [61] & E & $\begin{array}{c}57 \text { students } \\
\text { 1st-3rd Grade } \\
\text { (20 students in NIM, } \\
19 \text { in RRMF, } 18 \text { in } \\
\text { Control) }\end{array}$ & $\begin{array}{l}\text { (1) NIM only } \\
\text { (2) Repeated } \\
\text { Reading with } \\
\text { Multiple Features }\end{array}$ & 2) $R R+N I M$ & $\begin{array}{l}\text { Interventional level text (one } \\
\text { year above student's reading } \\
\text { level). } \\
\text { NIM } \\
\text { Instructional Steps: } \\
\text { (1) Teacher and student read } \\
\text { aloud with the teacher reading } \\
\text { slightly ahead of the student. } \\
\text { RR+NIM } \\
\text { Instructional Steps: } \\
\text { (1) Teacher and student read a } \\
\text { page or paragraph aloud with } \\
\text { the teacher reading slightly } \\
\text { ahead of the student; } \\
\text { (2) Student rereads the page or } \\
\text { paragraph aloud independently; } \\
\text { (3) Repeat steps until time is } \\
\text { complete. }\end{array}$ & $1: 1$ & $\begin{array}{c}7 \text { weeks } \\
20 \text { min. } \\
3 \text { days/week }\end{array}$ & $\begin{array}{c}\text { ORF: } \\
\text { Badar Reading and Language } \\
\text { Inventory: RRMF > CG (es = 0.06); } \\
\text { NIM > CG (es = 0.12) } \\
\text { MFS: } \\
\text { RRMF > CG (es = 1.16); NIM > CG (es } \\
=0.72) ; \text { RRMF = NIM } \\
\text { RC: } \\
\text { Badar Reading and Language } \\
\text { Inventory: } \\
\text { Retell: RRMF > CG (es = 1.47); NIM = } \\
\text { CG; RRMF = CG } \\
\text { Comprehension Questions: RRMF > } \\
\text { CG (es = 0.77); NIM > CG (es = 0.93); } \\
\text { RRMF = NIM }\end{array}$ \\
\hline
\end{tabular}

Note. $\mathrm{E}=$ Experimental; $\mathrm{QE}=$ Quasi-Experimental; $\mathrm{RRMF}=$ Repeated Reading with Multiple Features; NIM = Neurological Impress Method; $\mathrm{CR}=\mathrm{C}$ Continuous Reading; $\mathrm{LWR}=$ Listening While Reading; $\mathrm{LO}=$ Listening Only; CG = Control Group; ChR =Choral Reading; MFR = Model of Fluent Reading; EC = Error Correction; PD = Phrase Drill; PF = Performance Feedback; VC = Verbal Cues; GS = Goal Setting; ER = Echo Reading; LPP = Listening Passage Preview; $Q A$ = Question Answering; RR = Repeated Reading; ORF = Oral Reading Fluency; RC = Reading Comprehension; DORF = DIBELS Oral Reading Fluency; GORT-F = Gray Oral Reading Test-Fluency; GORT-C = Gray Oral Reading Test-Comprehension; QRI = Qualitative Reading Inventory; WRMT-PC = Woodcock Reading Mastery Test-Passage Comprehension; $C R A B=$ Comprehensive Reading Assessment Battery; MFS = Multidimensional Fluency Scale. For a description of multiple features, see Section 3.2.1 Repeated Reading. 


\subsection{Calculation of Effect Sizes}

All 16 studies included in this review provided adequate statistical information (i.e., sample means, pre- and post-intervention group means and standard deviations) that allowed us to calculate the effect sizes for the differences between the intervention and control groups on oral reading fluency outcomes. Nine of the studies provided statistical information to calculate effect sizes associated with reading comprehension outcomes. We chose to use the effect size formula by Carlson and Schmidt [62], where the difference between the intervention and control group pre-post-change mean score was divided by the pooled standard deviation of the intervention and control groups at pre-test. To reduce bias in the estimation of effect sizes, we also applied a bias correction parameter (that took into account the sample size the two groups) to the formula, as recommended in Morris [63]. This formula was chosen because (1) it accounted for pre-test differences between the intervention and control groups, (2) used a pooled standard deviation of both groups at pre-test only that reduced the potential of violating the homogeneity of variance assumption, and (3) findings from simulation studies showing the robustness of this method of effect size estimation [63]. Based on recommendations by Cohen [64], estimates of $0.20-0.49$ were considered small effects, $0.50-0.79$ medium effects, and values above 0.80 , large effects.

\section{Results}

\subsection{Participant Characteristics}

In the 16 studies reviewed, a total of 1112 students were included-506 second grade, 207 third grade, 120 fourth grade, and 76 fifth grade. Additionally, 57 participants were identified as primary grade students and 139 were identified as upper elementary students but further grade classification was undeterminable, and seven students were enrolled in sixth through eighth grade. The studies included 460 males, 419 females, and 233 students whose gender was unidentified. Ethnicity was reported for $75.5 \%$ of the students. Of those reported, 304 were African American, 221 were Caucasian, 208 were Hispanic, 63 were Asian, and 44 were mixed races or other. Across studies, the number of English Language Learners (ELLs) were reported in seven papers [48,50,54,55,57-59], with 295 students identified as ELLs. The number of students serviced through special education was also reported in seven studies [49,54-59]. A total of 140 students were identified as receiving special education services.

\subsection{Effective Oral Reading Fluency Interventions}

The 16 studies included in this review showed that repeated reading with multiple features (e.g., choral reading, verbal cueing, error correction) was most often empirically examined in order to determine the effectiveness on improving the oral reading fluency for elementary students with reading difficulties. Assisted reading, continuous reading, and the neurological impress method were also examined to a lesser extent and often in comparison to a repeated reading condition and control group. Across the 16 studies, one study reported no statistically significant gains for oral reading fluency and effect sizes for rate and accuracy measures in the remaining 15 studies varied widely, with negligible to large effects found ( $E S=0.01$ to 1.18 ). Three studies measured prosody outcomes and all found large, significant effects $[51,60,61]$. Effect sizes for reading comprehension also varied across papers. Of the nine studies from which effect sizes for reading comprehension outcomes could be calculated, one study reported no statistically significant gains [58], and eight studies had negligible to large effects on reading comprehension $(E S=0.07-2.59)[47,48,51,54,55,57,59,61]$.

\subsubsection{Repeated Reading}

Fourteen $(87.5 \%)$ of the 16 studies reviewed included a repeated reading intervention [46-48,51-61]; however, none of the studies examined a stand-alone repeated reading procedure. All of the interventions described included supplementary components in addition to the repeated reading procedures. The multiple features seen across studies included (1) a peer coach who was a classmate 
identified as more capable and fluent reader, (2) teacher modeling in which the adult served as a model of fluent and prosodic reading, (3) phrase-drill error correction which had the student say the phrase from the text containing each error three times after correct pronunciation was modeled by the instructor after an initial reading, (4) error correction that provided students during or after reading with a missed word and had them repeat the word after the instructor, (5) performance feedback in which the teacher provided information on students' prosody after reading, (6) verbal cueing which provided students with a reminder such as "Read this story the best you can and as quickly as you can.", (7) goal setting which had students set a WCPM goal prior to reading, (8) echo reading which asked the student to mimic the fluent and expressive reading of the instructor, (9) choral reading in which both the teacher and students read the text aloud together in unison, (10) performance of text, (11) listening passage preview which allowed students to listen and follow along while an adult read the text aloud with expression, (12) question answering after reading to promote comprehension, (13) neurological impress method in which the teacher read aloud slightly ahead of the student, and (14) phonics or vocabulary instruction. The number of multiple features included in repeated reading interventions varied widely across studies (See Table 1).

One-on-one with the teacher. In seven of the studies $[47,48,53,54,56,60,61]$, students participated in one-on-one sessions of a repeated reading with multiple features intervention with an adult (e.g., teacher, paraprofessional, trained research assistant). With regard to oral reading fluency, two studies were found to have negligible effects on students' rate and accuracy by the authors [53,61]. The remaining five studies were found to have small to large effect sizes (i.e., 0.38 to 1.18) on standardized measures of oral reading fluency (i.e., Dynamic Indicators of Basic Early Literacy Skills Oral Reading Fluency, Gray Oral Reading Test-Fluency) $[47,48,54,56,60]$. Four studies included measures of reading comprehension and found moderate to large effect sizes (i.e., 0.70-1.47) [47,48,54,61].

Specifically, Begeny, Mitchell, Whitehouse, Harris, and Stage as well as Begeny, Ross, Greene, Mitchell, and Whitehouse provided one-on-one repeated reading intervention three times a week to second graders who were native speakers of English and English Language Learners, respectively [47,48]. In both studies, the intervention produced large effect sizes (1.18 and 0.95) on students' oral reading fluency, as well as moderate and large effects on reading comprehension ( $E S=0.70$ and 1.12), respectively.

Young, Mohr, and Rasinski, examined the effects of a hybrid intervention combining repeated reading and the Neurological Impress Method (NIM) with third through fifth graders [60]. Students in the hybrid intervention condition demonstrated a moderately significant increase in rate and accuracy $(E S=0.68)$. In contrast, Young, Pearce, Gomez, Christensen, Pletcher, and Fleming tested the same hybrid intervention on a group of first- through third-grade students [61]. While negligible effects on oral reading fluency were found in this sample $(E S=0.07)$, moderate to large effects were found for reading comprehension (ES $=0.77$ for comprehension questions, $E S=1.47$ for passage retell). In addition to the intervention group and the no intervention control group, Young et al. also included a NIM-only group [61]. The students in the NIM only group were not provided with the opportunity to reread the text. Similar to the hybrid intervention condition, no effects on students' rate and accuracy were found by the authors. Further, the authors reported that the NIM-only condition had a nonsignificant effect on the passage retell aspect of reading comprehension but improved students' ability to answer comprehension questions after reading, as evidenced by a large, statistically significant effect size $(E S=0.93)$.

O'Connor, White, and Swanson provided second- and fourth-grade students with a repeated reading intervention that included error correction from the teacher [54]. The intervention, which lasted 14 weeks, produced a moderate effect $(E S=0.53)$ on students' oral reading fluency and a moderate to large effect on reading comprehension (ES $=1.09$ on Woodcock Reading Mastery Test-Passage Comprehension and ES $=0.75$ on Gray Oral Reading Test-Comprehension).

Therrien, Wickstrom, and Jones used a repeated reading intervention with fourth- through eighth-grade students which included a series of instructional steps such as verbal cueing and error 
correction [56]. The intervention was found to produce a small effect size $(E S=0.38)$ on students' oral reading fluency.

Finally, Martens et al. examined an after-school fluency training program in which second and third graders received a repeated reading intervention provided by graduate and undergraduate students [53]. Based on grade level CBM probes, negligible effects on students' oral reading fluency were found ( $E S=0.14$ for 2nd graders on 2nd grade probe and 0.06 for 3rd graders on 3rd grade probe). While the authors investigated rate and accuracy two days after the intervention ended, this was only completed for the intervention group and consequently, not enough information was provided to be able to calculate effect sizes associated with between-group differences.

One-on-one with a peer coach. Three studies examined the use of a repeated reading intervention in which students worked one-on-one with a peer coach $[46,52,55]$. Two of the three studies produced large effects on oral reading fluency outcomes [50,54].

In both Algozzine, Marr, Kavel, and Dugan and Marr, Algozzine, Nicholson, and Dugan [46,52], students worked together for 10-12 min, three times a week for 36 weeks. While both Algozzine et al. and Marr et al. found students in the intervention group had statistically significant gains in oral reading fluency from pre-test to post-test, statistically significant gains were also found for students in the no-intervention control group although at a lower rate. However, in both Algozzine et al. and Marr et al., repeated reading with a peer coach was found to produce large effect sizes on students' oral reading fluency (effect size $=1.06$ and 1.12, respectively). While reading comprehension growth in both studies was measured through an assessment created and used by the participating school district, data was only reported for the intervention group. Therefore, no effect sizes associated with between-group differences could be calculated for reading comprehension in these studies.

Similarly, Saenz, Fuchs, and Fuchs investigated Peer-Assisted Learning Strategies (PALS), with 20 special education and 33 low-achieving third- through sixth-grade students from bilingual classrooms [55]. Low-achieving students in this study were those in the lowest quartile of the class rank based on classroom observations, previous scores on state competence exams, and district informal reading inventories. In the PALS intervention, students worked in pairs in order to complete three readings of a text. With regard to oral reading fluency, a small effect $(E S=0.33)$ was found for ELL students with learning disabilities who participated in the PALS program three times a week for 15 weeks. Conversely, the same intervention demonstrated a negligible effect (0.01) for ELL students who were identified as low achieving. Further, in terms of reading comprehension, both ELL students with learning disabilities as well as ELL students who were identified as low achieving had large, statistically significant gains ( $E S=1.15$ for ELL+LD and 0.83 for ELL+ low achieving).

Small group with a teacher. Four studies had students working with a teacher in small groups of peers with similar needs and negligible to small effects were found with regard to oral reading fluency and reading comprehension outcomes [51,57-59]. Kuhn examined the effects on oral reading fluency and reading comprehension of (1) a repeated reading intervention with multiple features, (2) a listening-only intervention, (3) a continuous reading intervention, and (4) a no-intervention control group [51]. After six weeks of intervention, Kuhn concluded that students in the repeated reading condition outperformed students in the listening-only condition as well as the control group, although calculated effect sizes were trivial ( $E S=0.17$ and 0.14 , respectively). Additionally, non-significant gains in reading comprehension were found in the repeated reading condition, the listening-only condition, and the control group.

Vadasy and Sanders examined the same repeated reading intervention, QuickReads, with secondand third-grade students, including English Language Learners and special education students, across multiple sites $[58,59]$. The only difference between the studies was that Vadasy and Sanders first examined the effects of the intervention delivered by a trained paraprofessional and later examined the effects of the intervention delivered by a classroom teacher $[58,59]$.

In both studies $[58,59]$, the intervention procedures first had the instructor complete a brief letter-sound practice with the students due to the large number of students in the study with low 
word reading skills. Next, throughout the repeated reading procedure, instructors provided students with error correction strategies for students' miscues as needed (e.g., encouraging the student to sound out the word, phoneme by phoneme, and then blend the sounds; helping the student to segment a multisyllable word and then put the parts together). Both studies found the repeated reading intervention, QuickReads, to produce a small effect size on students' oral reading fluency when delivered by a trained paraprofessional ( $E S=0.41$ and 0.32 , respectively) and a moderate effect when delivered by a classroom teacher $(E S=0.53)[58,59]$. While Vadasy and Sanders found that the intervention employed by a paraprofessional did not produce a statistically significant difference in reading comprehension over the control group [58], the researchers determined that QuickReads delivered by a classroom teacher produced a small effect $(E S=0.36)$ [59].

Lastly, Vadasy and Sanders also examined a repeated reading intervention which included 199 fourth- and fifth-grade students with reading difficulties [57]. However, differing from their other studies [58,59], this intervention began and ended sessions with vocabulary instruction rather than letter-sound practice and, consequently, no error correction strategies were provided to students as they read. At the conclusion of the study, the researchers found that there were no significant treatment effects for oral reading fluency. Further, the average oral reading fluency rate of students in the intervention group remained in the lowest quartile, based on Hasbrouck and Tindal's norms for fourth and fifth grade [65]. However, a small effect (0.35) was found for reading comprehension [57].

\subsubsection{Listening While Reading}

Two studies examined the effects of an intervention which had students individually listen along to an audiobook while reading $[49,50]$. Esteves and Whitten's study investigated the assisted reading intervention with 20 upper elementary students, all of whom were serviced through special education with individualized education plan goals in the area of reading [49]. The researchers found that the students in the intervention group demonstrated a larger increase in the number of words read correctly from pre-test to post-test as compared to students in the no-intervention control group. A moderate effect on students' rate and accuracy was found $(E S=0.64)$.

Friedland, Gilman, Johnson, and Demeke also investigated the effect of listening while reading using audiobooks on a group of 46 third-grade students-almost half of which were English language learners [50]. After four weeks of intervention, Friedland and colleagues concluded that students in the treatment group demonstrated a greater improvement in the number of words read correctly per minute than students in the control group. However, a negligible effect size of 0.07 was obtained from our calculations. Interestingly, neither study of listening while reading included a measure of students' reading comprehension. Thus, we were not able to examine whether this method of fluency intervention also increased students' reading comprehension.

\subsubsection{Continuous Reading}

A continuous reading intervention was examined in a one-on-one setting by $\mathrm{O}^{\prime}$ Connor et al. and a small group setting by Kuhn [51,54]. Rather than rereading the same text multiple times, students in the continuous reading condition read continuously for the entirety of the intervention session and completed a single reading of a different text during each intervention session. Both studies found that continuous reading produced moderate gains in students' oral reading fluency rate and accuracy as well as moderate-to-large gains in reading comprehension

In O'Connor et al.'s study [54], second- and fourth-grade students in the continuous reading condition read a text at their instructional level (88\%-94\% accuracy) one-on-one with the teacher. The researchers investigated this continuous reading condition against a one-on-one repeated reading intervention as well as a no-intervention control group. Students in both intervention conditions read from the same text, only differing on whether they read the same page repeatedly or read multiple pages without repeating. Based on oral reading fluency rate scores at the end of the study, the researchers found that students in the continuous reading condition significantly outperformed 
students in the no intervention control group, with moderate effect sizes found $(E S=0.58)$. Further, a moderate-to-large effect (ES $=0.71$ on the Woodcock Reading Mastery Test-Passage Comprehension and $\mathrm{ES}=0.95$ on the Gray Oral Reading Test-Comprehension) was also found for reading comprehension. Comparing the repeated-reading-intervention and no intervention groups, moderate and large effect sizes for differences in oral reading fluency and reading comprehension were obtained, respectively $(E S=0.53$ and 1.93), favoring the repeated reading condition. It is important to note, however, that students in the repeated-reading-intervention did not differ significantly from students in the continuous-reading-intervention on measures of oral reading fluency or reading comprehension.

Similarly, Kuhn included a continuous reading condition in her study of oral reading fluency interventions with 24 second-grade students [51]. Differing from O'Connor et al.'s study [54], students in Kuhn's continuous reading intervention engaged in small-group echo or choral reading of a text with the adult tutor. At the end of the study, Kuhn reported that the students in the continuous reading intervention group outperformed students in the listening-only condition as well as the no-intervention control group for fluency, with calculated effect sizes of 0.58 and 0.26 respectively. However, mirroring $\mathrm{O}^{\prime}$ Connor et al. [54], there was no statistically significant difference between students in the continuous reading and repeated reading conditions for oral reading fluency. Further, only students in the continuous reading condition demonstrated greater improvement in reading comprehension in comparison to the control group, and a large effect was obtained in the calculation of between-group differences $(\mathrm{ES}=2.59)$.

\subsection{Prosody}

While all of the studies reviewed provided measures of rate and accuracy, only three (18.8\%) studies reported measures of students' prosody [51,60,61]. Kuhn assessed students' prosody using the NAEP's Oral Reading Fluency Scale and reported 100\% interrater reliability between two raters on this measure [51,66]. Kuhn found that both intervention conditions (i.e., repeated reading and continuous reading) produced gains in students' prosody that were not seen in students who were in the listening-only condition or the no-intervention control group. Calculated effect sizes found large effects on prosody outcomes for both repeated reading and continuous reading in comparison to a control group (ES $=0.86$ and 1.28 , respectively).

Young and colleagues used the Multidimensional Fluency Scale (MFS), a prosody rubric which calculated a score in four distinct categories: (1) volume and expression, (2) phrasing, (3) smoothness, and (4) pace, in order to assess students' prosody [60,61]. Rasinski, Rikli, and Johnston previously demonstrated that the MFS was a reliable and valid measure of prosodic reading [67]. Across studies, the hybrid intervention of repeated reading and NIM produced large, significant effects on students' prosody (ES $=0.98$ and 1.16, respectively) [55,57]. Further, the intervention consisting of NIM alone also yielded a moderate effect size (0.72) for prosody [61].

\section{Discussion}

The primary objective of the present systematic review was to examine the effectiveness of oral reading fluency interventions on aspects of oral reading fluency (i.e., accuracy, rate, and prosody) and reading comprehension for children in first through fifth grades with reading difficulties. Additionally, we identified characteristics pertaining to the intervention format for studies where positive gains in reading outcomes were made after intervention in reading fluency was provided.

With regard to the first question on the effectiveness of oral language interventions on reading outcomes, out of the 16 studies examined in this review, 12 (75\%) were found to produce small to large effects on the oral reading fluency of students with reading difficulties. Further, eight (50\%) studies had small to large effects on reading comprehension outcomes for these students, with the majority of interventions yielding moderate to large effect sizes across a variety of measures. Broadly, these findings point to the efficacy of targeted fluency instruction in promoting reading comprehension, and to a lesser extent, oral reading fluency outcomes, among first to fifth graders with reading difficulties. 
In considering these results, we noted four interesting trends. First, the findings demonstrated that benefits of fluency interventions were not limited only to aspects of fluency that were the target of the interventions but also extended to reading comprehension outcomes. This finding is not surprising for two reasons. The first is that it is logical when we explain them within reading models that consider the role of fluency. Chall's stages of reading development outline the importance of fluency as a bridge that moves students from focusing on decoding to extracting meaning from connected texts [3,37-39]. Similarly, when we consider the theory of automatic word processing, oral reading fluency interventions provide students with practice in reading connected texts that help students gain automaticity with word recognition and thus devote more cognitive resources to comprehension [11]. Second, many of the studies reviewed reported having students engage in summarizing, generating questions, and retelling texts that they have read, which are activities included in reading comprehension interventions that help struggling students [68,69]. Further, since many of the interventions reviewed also included re-reading of texts (a fluency building activity) as a central element, our findings also suggest the utility of including fluency-building elements in interventions aimed at improving reading comprehension. This is aligned with research suggesting that multifaceted interventions that incorporate the teaching of a variety of reading-related skills are more effective than those that target only one skill $[70,71]$. Future research could investigate oral reading fluency interventions in comparison to interventions directly targeting reading comprehension for both fluency and reading comprehension outcomes. This may help to provide teachers with greater insight on the most effective interventions for students with reading difficulties.

The second trend is that effect sizes yielded for reading comprehension outcomes were on average larger than that for oral reading fluency. Additionally, there was a certain degree of heterogeneity of magnitude of effect sizes across studies on both outcomes, although the heterogeneity appeared to be more salient in oral reading fluency outcomes. Considering that the studies reviewed in this present synthesis examined reading fluency interventions, outcome measures of oral reading fluency would be considered more proximal outcomes as compared to reading comprehension. Therefore, this trend is somewhat surprising since it has been shown that effects of reading interventions on proximal outcomes are often greater than distal outcomes [72,73]. We put forth two possible explanations for this trend, both attributable to the method of measurement. Current conceptualizations of reading fluency favor the view that fluency encompasses both decoding and comprehension, as opposed to just decoding. Most studies reviewed assessed oral reading fluency using the Dynamic Indicators of Basic Early Literacy Skills (DIBELS), which functions more as a screening tool that focuses primarily on accuracy and speed of decoding rather than a comprehensive measure of reading skills [74]. Because inconsistencies in the association between fluency subtests of the DIBELS have been reported in some studies [75], researchers have suggested that the use of one-minute probes in DIBELS might not adequately capture all aspects of reading fluency [76]. This could explain why reading comprehension outcomes showed greater effect sizes as compared to oral reading fluency. On a related note, it was observed that there were more instances of the use of informal measures of oral reading fluency as compared to reading comprehension in the studies reviewed and most of these measures yielded increases of negligible effects for rate and accuracy outcomes. A major concern with the use of informal reading measures relates to the reliability of these measures [77], therefore, this trend calls for future studies to examine the effects of interventions on both informal and formal measures of fluency in order to gain a better picture of the extent of impact of fluency interventions.

The third trend is that outcome measures of fluency used to assess the efficacy of the interventions focused on accuracy and rate aspects in most of the studies reviewed. This is predictable considering that these two components are widely regarded as representative components of fluency [10]. In contrast, only three studies documented interventions that examined or included prosody measures. This is despite the fact that prosody is also highlighted as a component of fluency in both the NRP and the Common Core State Standards (CCSS) as well as by prominent scholars in the field $[5,78,79]$. The lack of attention to prosody has been highlighted by researchers [22]. We put forth two reasons for 
why this third component is relatively neglected as compared to the other two aspects. First, there have been issues surrounding the accuracy of measurement of this component. Prosody encompasses several subcomponents such as pitch, intonation, and phrasing and is typically assessed using rating scales [80]. However, researchers have pointed out that rating scales might not adequately differentiate between readers of varying levels of fluency [81]. Second, the assessment of prosody is often viewed as being subjective based on the views of the person completing the assessment, which raises questions about the validity and reliability issues in assessing prosody [82]. In the present study, all three studies reported in this study showed positive increases in prosody following intervention, although different measures and aspects of prosody were measured across studies. However, the dearth of literature on prosody and variability in measurement of prosody makes it difficult to draw definite conclusions on the effects of interventions on prosody. This gap points to the need for future research to assess this aspect more systematically and comprehensively.

The fourth trend is that close to $90 \%$ of the studies reviewed in this search examined the same type of intervention, repeated reading. This focus on repeated reading in the literature is not surprising considering the strong theoretical underpinnings of this strategy $[83,84]$, leading to researchers and educators alike viewing it favorably as an evidence-based approach to improving fluency [43]. This trend concurs with that in the synthesis conducted by Kim, Bryant, Bryant, and Park who found that $75 \%$ of the studies reviewed focused on repeated reading interventions for students with learning disabilities [45]. Our findings lend further support to the efficacy of this type of reading intervention for children with reading difficulties in promoting reading outcomes (see [39] for a review). They also provide further justification for the continued use of repeated reading in building up fluency. Repeated reading is effective because it addresses at least two of the three main problems that lead to dysfluent reading as identified in theoretical frameworks. These three main problems pertain to (1) difficulty with identifying prosodic cues, (2) labored word recognition, and (3) difficulty making associations between meanings and main ideas in text [85]. Repeated reading of text promotes practice with reading which facilitates speed and ease in the recognition of words/texts. This in turn, leads to more working memory resources being freed up, which according to Samuels and Laberge's model of automaticity in reading, allows one to focus on higher-order processes pertaining to comprehension such as accessing meaning of words and building up a situation model of the text [15]. Furthermore, at the level of higher-order processing, successive readings of the same text allow readers to focus on different pieces of information in the text across readings and improve memory of central ideas that facilitate comprehension [86]. However, despite the success of the repeated reading method in promoting fluency and reading outcomes across studies, researchers also point out that specific characteristics of interventions impact the extent in which fluency interventions are effective $[38,39,45,87,88]$. Our second research objective thus focused on intervention characteristics pertaining to (1) instructional variables (e.g., pre-instructional support, the difficulty level of text used, the number of times the text is read, and the amount of feedback provided), and (2) type of interventionist (e.g., teacher, clinician, researcher, parent, or peer coach) and how they influenced intervention outcomes.

It would appear that by examining effect sizes only across studies that there was no clear pattern of how type of interventionist relates to effectiveness of fluency interventions. This is as interventions across studies that were facilitated by either an adult or a peer coach yielded similarly positive effects on oral reading fluency outcomes as interventions that were facilitated by both an adult or a peer coach during the school day tended to yield positive effects on oral reading fluency outcomes. However, a closer examination of characteristics of studies suggests the need to consider a combination of other factors when evaluating effect sizes relating to type of interventionist. The first factor is whether the interventionist received appropriate and adequate training. We note that across studies, training was always provided to both the adults and students prior to the start of the intervention. For example, in Begeny and colleague's study which yielded sizeable effect sizes, it was highlighted that the teachers participating in the repeated reading intervention were provided with two 3-hour training sessions. In these sessions, they observed a trainer modeling the intervention procedures and were provided with 
time to practice implementing the intervention with one another under guidance from the research staff $[47,48]$. Training such as this may be key to the success of the oral reading fluency intervention since other studies show reading interventions conducted by trained tutors have been shown to be more effective than untrained tutors [89].

The second factor to consider is the close association between decoding (i.e., phonemic awareness, letter-sound correspondences) and fluency skills. It was observed that in three of the studies where a trained teacher facilitated repeated reading intervention, no effects on students' rate and accuracy outcomes were found. The authors across these studies made mention of issues pertaining to decoding skills in accounting for these null findings. For instance, Vadasy and Sanders attributed their non-significant findings to the use of a vocabulary extension activity rather than explicit instruction in alphabetic knowledge and decoding strategies in the intervention [57], where the latter was considered as being more crucial to developing automaticity in reading [11]. Similarly, Martens et al. acknowledged that the second-grade students in their sample may have yet to master the foundational reading skills (i.e., phonemic awareness, sight word vocabulary) necessary for fluency-building interventions to be effective [53]. The importance of considering decoding elements in addition to fluency building activities in fluency interventions echoes the views of researchers in the field [85,90]. Future research that directly compares interventions with and without a focus on decoding and/or considers decoding abilities of children before and after interventions would provide insights into the significance of including decoding instruction to fluency interventions.

In thinking about group size, intervention provided by an adult in a one-on-one setting appeared to be more effective than interventions utilizing a small-group format. Nine of the 16 studies reviewed provided an oral reading fluency intervention in a one-on-one setting with an adult $[47,48,53,54,56,60,61]$. The majority $(56 \%)$ of studies that employed a one-on-one student-to-teacher ratio for interventions were found to produce moderate to large effect sizes (ES $=0.53-1.18$ for oral reading fluency and ES = 0.70-1.47 for reading comprehension) whereas the remainder of studies yielded small or negligible effects for oral reading fluency rate and accuracy. The variability in effect sizes across studies should be discussed in the context of other intervention characteristics. For one, previous research has indicated that the inclusion of more fluency-building features in instruction promoted better outcomes [39]. An examination of the magnitude of effect sizes across studies with one-on-one formats appear to support this. For instance, Begeny and colleagues' two studies which yielded large effect sizes on oral reading fluency measures $[47,48]$, incorporated a total of seven additional features-six of which targeted aspects of fluency including prosody (i.e., teacher modeling, performance feedback, verbal cueing), accuracy (i.e., phrase-drill error correction), and motivation (i.e., goal setting, reward system). Conversely, in Therrien et al. [56], where a small effect was found, only three of the five features in the intervention focused on building fluency (i.e., verbal cueing, goal setting, error correction).

Interestingly, across studies, the repeated reading intervention often included a model of fluent reading or accurate word pronunciation (e.g., error correction, phrase drill). Chard et al. highlighted that repeated reading with a model is often more effective at increasing students' oral reading fluency and reading comprehension [38]. However, our review showed that some studies with negligible or small effects on oral reading fluency also included modeling procedures. For example, Martens et al. examined an after-school intervention program which included modeling through listening passage preview and phrase drill error correction [53]. While negligible effects were found for students oral reading fluency, it is unclear if absenteeism and attrition impacted the results as these issues have plagued other after school intervention studies [91]. This suggests the need for future research to examine the context in which these modeling strategies are most effective.

In addition, intervention duration also appears to affect the effectiveness of different types of one-on-one interventions. To illustrate, while Esteves and Whitten found a moderate effect for a listening while reading intervention $(E S=0.64)$ [49], Friedland et al.'s intervention of the same nature was found to have no effect [50]. When examining the two studies, the duration of intervention was vastly different, with students in Esteves and Whitten's study spending three to six times longer in the 
intervention than students in Friedland et al.'s study. Conversely, the duration of the intervention did not appear to contribute to the effectiveness of the repeated reading interventions examined. This suggests that the success of a listening while reading intervention, but not repeated reading, might be partly dependent on duration of intervention. However, we recognize that the available sample of studies for such a comparison is small in the present review, and thus this hypothesis necessitates further validation.

In contrast to one-on-one interventions, three of the four studies with small-group format interventions yielded negligible or small effects in fluency and/or reading comprehension [57-59]. The only exception was the study by Kuhn [51] where a large effect size was found for reading comprehension outcomes. Although the findings appear to support one-on-one interventions, a practical constraint to consider is that this format is time consuming and classroom teachers may not be able to devote the time needed to provide one-on-one interventions. This is especially true if the classroom has a high percentage of students with or at risk for reading difficulties. One viable option may be the use of peer tutors, as two studies of peer coaching in this review produced large and significant effects for oral reading fluency [46,52]. However, these findings should also be interpreted with caution since three of the four studies on small groups and two of the three studies on peer coaches came from the same research teams, respectively. In addition, we note that there was substantial heterogeneity in the features of the intervention between studies that conducted small-group interventions as opposed to those that carried out interventions in one-on-one formats. Therefore, future research examining small-group oral reading fluency interventions, especially in direct comparison to one-on-one interventions, may be beneficial in informing classroom practices.

\section{Limitations and Future Research}

This review should be considered in the context of its limitations. One limitation is that conclusions about reading comprehension should be interpreted with caution since a wide variety of reading comprehension measures were used across studies. Future research is encouraged to utilize common standardized measures of reading comprehension so that results across studies may be compared in a more meaningful manner.

Furthermore, one important question posed by Chard et al. is still left unanswered [38]. Chard et al. suggested that future researchers investigate whether the effects of fluency building interventions are sustainable over time. In the present synthesis, only one study included a measure of retention which assessed students' oral reading fluency two days post-intervention. Future research may also wish to examine the long-term maintenance of oral reading fluency gains found in students who received a successful fluency intervention, a limitation acknowledged by Martens and colleagues [53]. In addition, the findings of this synthesis also point to gaps in our understanding of the efficacy of interventions other than repeated reading and on prosody outcomes. Future studies should examine other commonly used interventions such as choral and echo reading or readers' theater and examine prosody outcomes in the assessment of fluency in order to provide a more comprehensive understanding of fluency instruction and reading. Finally, researchers have acknowledged that the increasing attention to fluency in the US context can be attributed in large part to its inclusion in the National Reading Panel report published in 2000 [5]. This could explain why studies yielded for this and previous reviews were conducted primarily in the U.S. context despite no country filters being imposed in the search. The lack of diversity in this respect points to the need for more studies in other contexts where differences in instructional curriculum might exist in order to understand whether and how instructional curriculum might moderate the impact of fluency interventions. This is especially important considering that fluency is a central component in curriculum standards in many countries and languages [6].

\section{Implications and Conclusions}

Despite its limitations, the present synthesis contributes to the literature in three ways. First, our findings support previous syntheses- the last of which was conducted by Stevens et al. with studies 
between 2001 and 2014 [40]. Generally, fluency interventions examined, which constituted mostly repeated reading procedures, made positive contributions to gains in reading fluency and reading comprehension among children with reading difficulties. Considering that failure to replicate findings presents as a salient issue in research [92,93], the replication of findings of previous syntheses despite not including single-case studies and only focusing on those studies that included a control group provides convergent evidence for the importance of fluency on reading outcomes. Second, extending from previous syntheses, our finding compared relative gains of fluency interventions on fluency and reading comprehension outcomes. The observation that fluency interventions produced greater gains in comprehension as compared to fluency in general adds credence to the current view that reading fluency relates to both decoding and comprehension rather than the earlier conceptualization that fluency was confined mainly to word recognition $[5,94]$. Third, the current study also examined characteristics of interventions and their impact on reading outcomes, including the impact of group size in intervention, a characteristic that had not been systematically investigated in previous studies. Our findings thus have implications on instructional practices in the classroom.

The findings from this synthesis suggest that elementary students with reading difficulties would benefit from one-on-one interventions which include a model of fluent reading who has received adequate training. The findings support earlier research which suggests that the repeated reading of text is one way to build the oral reading fluency of students with reading difficulties. However, continuous reading with teacher support may also be an effective method. Moreover, although listening while reading and small-group interventions produced only small to moderate effect sizes, they may be more efficient means of improving oral reading fluency since they require less instructional time of the classroom teacher. However, an important fact to remember about any intervention, and one Young et al. also mentioned, is that there is not one intervention that is effective for every student and if not responding to one type of fluency intervention, some students may need an alternate form of instruction [60].

Research on the efficacy of certain approaches to teaching fluency has led to changes in instructional practices and should continue to do so [95]. With more recent focus on Response to Intervention (RTI) and Multi-Tiered Systems of Support (MTSS), evidence-based fluency strategies should be used to target students with and at risk for reading difficulties in classroom instruction, as well as in small-group intervention outside of the classroom. Listening while reading, repeated reading with multiple features, and continuous reading with teacher support are methods that can be effectively used across a variety of contexts for increasing the oral reading fluency of elementary students who need more targeted instruction.

Author Contributions: Conceptualization, A.H. and E.B.-C.; Methodology, A.H., P.W.K., and K.A.M.; Investigation, A.H., P.W.K., and K.A.M.; Writing-Original Draft Preparation, A.H., P.W.K., and K.A.M.; Writing-Reviewing \& Editing: A.H., P.W.K., K.A.M., and E.B.-C. All authors have read and agreed to the published version of the manuscript.

Funding: This research received no external funding.

Conflicts of Interest: The authors declare no conflict of interest.

\section{References}

1. National Assessment of Educational Progress. The Nation's Report Card: Reading 2019; National Center for Educational Statistics: Washington, DC, USA, 2019.

2. Lerner, J. Learning Disabilities: Theories, Diagnosis, and Teaching Strategies; Houghton Mifflin: New York, NY, USA, 2003.

3. Chall, J.S. Stages of Reading Development; McGraw-Hill: New York, NY, USA, 1983.

4. Daane, M.C.; Campbell, J.R.; Grigg, W.S.; Goodman, M.J.; Oranje, A. Fourth-Grade Students Reading Aloud: NAEP 2002 Special Study of Oral Reading; (NCES 2006-469); U.S. Department of Education, Institute of Education Sciences, National Center for Education Statistics: Washington, DC, USA, 2005. 
5. National Reading Panel (US); National Institute of Child Health and Human Development (US). Teaching Children to Read: An Evidence-Based Assessment of the Scientific Research Literature on Reading and Its Implications for Reading Instruction; National Institute of Child Health and Human Development, National Institutes of Health: Rockville, MD, USA, 2000.

6. Abadzi, H. Reading Fluency Measurements in EFA FTI Partner Countries: Outcomes and Improvement Prospects; Tech. Rep., Global Partnership for Education; World Bank: Washington, DC, USA, 2011.

7. Wimmer, H.; Mayringer, H.; Landerl, K. Poor reading: A deficit in skill-automatization or a phonological deficit? Sci. Stud. Read. 1998, 2, 321-340. [CrossRef]

8. de Jong, P.F.; van der Leij, A. Developmental changes in the manifestation of a phonological deficit in dyslexic children learning to read in a normally achieving orthography. J. Educ. Psychol. 2003, 95, 22-40. [CrossRef]

9. Kuhn, M.R.; Rasinski, T.; Young, C. The best practices in fluency instruction. In Best Practices in Literacy Instruction, 6th ed.; Morrow, L.M., Gambrell, L.B., Eds.; Guilford Press: New York, NY, USA, 2018; pp. 271-288.

10. Kuhn, M.R.; Stahl, S.A. Fluency: A review of developmental and remedial practices. J. Educ. Psychol. 2003, 95, 3-21. [CrossRef]

11. Gough, P.; Tunmer, W. Decoding, reading, and reading disability. Rem. Spec. Educ. 1986, 7, 6-10. [CrossRef]

12. Allington, R.L. Fluency: The neglected reading goal. Read. Teach. 1983, 36, 556-561.

13. Scarborough, H.S. Connecting early language and literacy to later reading (dis)abilities: Evidence, theory, and practice. In Handbook for Research in Early Literacy; Neuman, S., Dickinson, D., Eds.; Guilford Press: New York, NY, USA, 2001; pp. 97-110.

14. Kim, Y.S.G.; Wagner, R.K. Text (oral) reading fluency as a construct in reading development: An investigation of its mediating role for children from grades 1 to 4. Sci. Stud. Read. 2015, 19, 224-242. [CrossRef] [PubMed]

15. LaBerge, D.; Samuels, S.J. Toward a theory of automatic information processing in reading. Cognit. Psychol. 1974, 6, 293-323. [CrossRef]

16. Cain, K.; Oakhill, J.; Bryant, P. Children's reading comprehension ability: Concurrent prediction by working memory, verbal ability, and component skills. J. Educ. Psychol. 2004, 96, 31-42. [CrossRef]

17. Palladino, P.; Cornoldi, C.; DeBeni, R.; Pazzaglia, F. Working memory and updating processes in reading comprehension. Mem. Cognit. 2001, 29, 344-354. [CrossRef] [PubMed]

18. Garnett, K. Fluency in learning to read: Conceptions, misconceptions, learning disabilities, and instructional moves. In Multisensory Teaching of Basic Language Skills, 3rd ed.; Birsh, J.R., Ed.; Paul H. Brookes Publishing Co.: Baltimore, MD, USA, 2011.

19. Silverman, R.D.; Speece, D.L.; Harring, K.R.; Ritchey, K.D. Fluency has a role in the simple view of reading. Sci. Stud. Read. 2012, 17, 108-133. [CrossRef]

20. O'Connor, R.E. Reading fluency and students with reading disabilities: How fast is fast enough to promote reading comprehension? J. Learn. Disabil. 2018, 51, 124-136. [CrossRef] [PubMed]

21. Sabatini, J.P.; O'Reilly, T.; Halderman, L.K.; Bruce, K. Integrating scenario-Based and component reading skill measures to understand the reading behavior of struggling readers. Learn. Disabil. Res. Pr. 2014, 29, 36-43. [CrossRef]

22. Rasinski, T.V. Reading fluency instruction: Moving beyond accuracy, automaticity, and prosody. Read. Teach. 2006, 59, 704-706. [CrossRef]

23. Zutell, J.; Donelson, R.; Mangelson, J.; Todt, P. Building a focus on oral reading fluency into individual instruction for struggling readers. In Fluency Instruction: Research-Based Best Practices, 2nd ed.; Rasinski, T., Blachowicz, C., Lems, K., Eds.; Guildford Press: New York, NY, USA, 2012.

24. Miller, J.; Schwanenflugel, P.J. Prosody of syntactically complex sentences in the oral reading of young children. J. Educ. Psychol. 2006, 98, 839-843. [CrossRef]

25. Meisinger, E.; Bloom, J.; Hynd, G. Reading fluency: Implications for the assessment of children with reading disabilities. Ann. Dyslexia 2010, 60, 1-17. [CrossRef]

26. Individuals with Disability Education Act [IDEA]. Available online: http://www.p12.nysed.gov/specialed/ idea/108-446.pdf (accessed on 26 September 2019).

27. Archer, A.L.; Gleason, M.M.; Vachon, V.L. Decoding and fluency: Foundation skills for struggling older readers. Learn. Disabil. Q. 2003, 26, 89-101. [CrossRef]

28. Rasinski, T.V.; Padak, N.D. How elementary students referred for compensatory reading instruction perform on school-based measures of word recognition, fluency, and comprehension. Read. Psychol. 1998, 19, 185-216. [CrossRef] 
29. Kang, E.Y.; Shin, M. The contributions of reading fluency and decoding to reading comprehension for struggling readers in fourth grade. Read. Writ. Q. 2019, 35, 179-192. [CrossRef]

30. Lovett, M.W.; Steinbach, K.A.; Frijters, J.C. Remediating the core deficits of developmental reading disability: A double-deficit perspective. J. Learn. Disabil. 2000, 33, 334-358. [CrossRef]

31. Just, M.A.; Carpenter, P.A. A capacity theory of comprehension: Individual differences in working memory. Psychol. Rev. 1992, 99, 122-149. [CrossRef]

32. Logan, G.D. Automaticity and reading: Perspectives from the instance theory of automatization. Read. Writ. Q. 1997, 13, 123-146. [CrossRef]

33. Swanson, H.L.; Zheng, X.; Jerman, O. Working memory, short-term memory, and reading disabilities: A selective meta-analysis of the literature. J. Learn. Disabil. 2009, 42, 260-287. [CrossRef] [PubMed]

34. Begeny, J.C.; Laugle, K.M.; Krouse, H.E.; Lynn, A.E.; Tayrose, M.P.; Stage, S.A. A control-group comparison of two reading fluency programs: The Helping Early Literacy with Practice Strategies (HELPS) program and the great leaps K-2 reading program. Sch. Psychol. Rev. 2010, 39, 137-155.

35. Lyon, G.R.; Moats, L.C. Critical conceptual and methodological considerations in reading intervention research. J. Learn. Disabil. 1997, 30, 578-588. [CrossRef]

36. Wexler, J.; Vaughn, S.; Edmonds, M.; Reutebuch, C.K. A synthesis of fluency interventions for secondary struggling readers. Read. Writ. 2008, 21, 317-347. [CrossRef]

37. Lee, J.; Yoon, S.Y. The effects of repeated reading on reading fluency for students with reading disabilities: A meta-analysis. J. Learn. Disabil. 2017, 50, 213-224. [CrossRef]

38. Chard, D.J.; Vaughn, S.; Tyler, B.J. A synthesis of research on effective interventions for building fluency with elementary students with learning disabilities. J. Learn. Disabil. 2002, 35, 386-406. [CrossRef]

39. Therrien, W.J. Fluency and comprehension gains as a result of repeated reading. Rem. Spec. Educ. 2004, 24, 252-261. [CrossRef]

40. Stevens, E.A.; Walker, M.A.; Vaughn, S. The effects of reading fluency interventions on the reading fluency and reading comprehension performance of elementary students with learning disabilities: A synthesis of the research from 2001 to 2014. J. Learn. Disabil. 2017, 50, 576-590. [CrossRef]

41. Elbaum, B.; Vaughn, S.; Hughes, M.; Moody, S.W. Grouping practices and reading outcomes for students with disabilities. Except. Child. 1999, 65, 399-415. [CrossRef]

42. Torgesen, J.K.; Alexander, A.W.; Wagner, R.K.; Rashotte, C.A.; Voeller, K.K.S.; Conway, T. Intensive remedial instruction for children with severe reading disabilities: Immediate and long-term outcomes from two instructional approaches. J. Learn. Disabil. 2001, 34, 33-58. [CrossRef] [PubMed]

43. Chard, D.; Ketterlin-Geller, L.; Baker, S.; Doabler, C.; Apichatabutra, C. Repeated reading interventions for students with learning disabilities: Status of the evidence. Except. Child. 2009, 75, 263-281. [CrossRef]

44. Garnett, K. Fluency in learning to read: Conceptions, misconceptions, learning disabilities, and instructional moves. In Multisensory Teaching of Basic Language Skills, 4th ed.; Birsh, J.R., Carreker, S., Eds.; Paul H. Brookes Publishing Co.: Baltimore, MD, USA, 2018.

45. Kim, M.K.; Bryant, D.P.; Bryant, B.R.; Park, Y. A synthesis of interventions for improving oral reading fluency of elementary students with learning disabilities. Prev. Sch. Fail. 2017, 61, 116-125. [CrossRef]

46. Algozzine, B.; Marr, M.B.; Kavel, R.L.; Dugan, K.K. Using peer coaches to build oral reading fluency. J. Educ. Stud. Pl. R. 2009, 14, 256-270. [CrossRef]

47. Begeny, J.C.; Mitchell, R.C.; Whitehouse, M.H.; Harris, C.F.; Stage, S.A. Effects of the HELPS reading fluency program when implemented by classroom teachers with low-performing second-grade students. Learn. Disabil. Res. Pr. 2011, 26, 122-133. [CrossRef]

48. Begeny, J.C.; Ross, S.G.; Greene, D.J.; Mitchell, R.C.; Whitehouse, M.H. Effects of the Helping Early Literacy with Practice Strategies (HELPS) reading fluency program with Latino English language learners: A preliminary evaluation. J. Behav. Educ. 2012, 21, 134-149. [CrossRef]

49. Esteves, K.J.; Whitten, E. Assisted reading with digital audiobooks for students with reading disabilities. Read. Horizons 2011, 51, 21-40.

50. Friedland, A.; Gilman, M.; Johnson, M.; Demeke, A. Does reading-while-listening enhance students' reading fluency? Preliminary results from school experiments in rural Uganda. J. Educ. Pract. 2017, 8, 82-95.

51. Kuhn, M. A comparative study of small group fluency instruction. Read. Psychol. 2005, 26, 127-146. [CrossRef] 
52. Marr, M.B.; Algozzine, B.; Nicholson, K.; Dugan, K.K. Building oral reading fluency with peer coaching. Rem. Spec. Educ. 2011, 32, 256-264. [CrossRef]

53. Martens, B.K.; Eckert, T.L.; Begeny, J.C.; Lewandowski, L.J.; DiGennaro, F.D.; Montarello, S.A.; Arbolino, L.A.; Reed, D.D.; Fiese, B.H. Effects of a fluency-building program on the reading performance of low-achieving second and third grade students. J. Behav. Educ. 2007, 16, 39-54. [CrossRef]

54. O'Connor, R.E.; White, A.; Swanson, H.L. Repeated reading versus continuous reading: Influences on reading fluency and comprehension. Except. Child. 2007, 74, 31-46. [CrossRef]

55. Saenz, L.M.; Fuchs, L.S.; Fuchs, D. Peer-assisted learning strategies for English language learners with learning disabilities. Except. Child. 2005, 71, 231-247. [CrossRef]

56. Therrien, W.J.; Wickstrom, K.; Jones, K. Effect of a combined repeated reading and question generation intervention on reading achievement. Learn. Disabil. Res. Pr. 2006, 21, 89-97. [CrossRef]

57. Vadasy, P.F.; Sanders, E.A. Benefits of repeated reading intervention for low-achieving fourth- and fifth-grade students. Rem. Spec. Educ. 2008, 29, 235-249. [CrossRef]

58. Vadasy, P.F.; Sanders, E.A. Repeated reading intervention: Outcomes and interactions with readers' skills and classroom instruction. J. Educ. Psychol. 2008, 100, 272-290. [CrossRef]

59. Vadasy, P.F.; Sanders, E.A. Supplemental fluency intervention and determinants of reading outcomes. Sci. Stud. Read. 2009, 13, 383-425. [CrossRef]

60. Young, C.; Mohr, K.A.J.; Rasinski, T. Reading together: A successful reading fluency intervention. Lit. Res. Instr. 2015, 54, 67-81. [CrossRef]

61. Young, C.; Pearce, D.; Gomez, J.; Christensen, R.; Pletcher, B.; Fleming, K. Read two impress and the neurological impress method: Effects on elementary students' reading fluency, comprehension, and attitude. J. Educ. Res. 2018, 111, 657-665. [CrossRef]

62. Carlson, K.D.; Schmidt, F.L. Impact of experimental design on effect size: Findings from the research literature on training. J. App. Psychol. 1999, 84, 851-862. [CrossRef]

63. Morris, S.B. Estimating effect sizes from pretest-posttest-control group designs. Organ. Res. Methods 2008, 11, 364-386. [CrossRef]

64. Cohen, J. Statistical Power Analysis for the Behavioral Sciences, 2nd ed.; Lawrence Earlbaum Associates: Hillsdale, NJ, USA, 1988.

65. Hasbrouck, J.; Tindal, G. Oral reading fluency norms: A valuable assessment tool for teachers. Read. Teach. 2006, 59, 636-644. [CrossRef]

66. U.S. Department of Education. NAEP's Oral Reading Fluency Scale; U.S. Department of Education, Office of Educational Research and Improvement: Washington, DC, USA, 1995.

67. Rasinski, T.; Rikli, A.; Johnston, S. Reading fluency: More than automaticity? More than a concern for the primary grades? Lit. Res. Instr. 2009, 48, 350-361. [CrossRef]

68. Edmonds, M.S.; Vaughn, S.; Wexler, J.; Reutebuch, C.; Cable, A.; Tackett, K.K.; Schnakenberg, J.W. A synthesis of reading interventions and effects on reading comprehension outcomes for older struggling readers. Rev. Educ. Res. 2009, 79, 262-300. [CrossRef]

69. Kamil, M.L. Vocabulary and comprehension instruction: Summary and implications of the national reading panel findings. In The Voice of Evidence in Reading Research; McCardle, P., Chhabra, V., Eds.; Paul H. Brookes: Baltimore, MD, USA, 2004.

70. Morris, R.D.; Lovett, M.W.; Wolf, M.; Sevcik, R.A.; Steinbach, K.A.; Frijters, J.C.; Shapiro, M.B. Multiple-component remediation for developmental reading disabilities: IQ, socioeconomic status, and race as factors in remedial outcome. J. Learn. Disabil. 2012, 45, 99-127. [CrossRef]

71. Hay, I.; Elias, G.; Fielding-Barnsley, R.; Homel, R.; Freiberg, K. Language delays, reading delays, and learning difficulties: Interactive elements requiring multidimensional programming. J. Learn. Disabil. 2007, 40, 400-409. [CrossRef]

72. Solis, M.; Ciullo, S.; Vaughn, S.; Pyle, N.; Hassaram, B.; Leroux, A. Reading comprehension interventions for middle school students with learning disabilities: A synthesis of 30 years of research. J. Learn. Disabil. 2012, 45, 327-340. [CrossRef]

73. Swanson, H.L.; Hoskyn, M.; Lee, C. Interventions for Students with Learning Disabilities; Guilford: New York, NY, USA, 1999. 
74. Good, R.H.; Gruba, G.G.; Kaminski, R.A. Best practices in using Dynamic Indicators of Basic Early Literacy Skills (DIBELS). In Best Practices in School Psychology IV; Thomas, A., Grimes, J., Eds.; National Association of School Psychologists: Washington, DC, USA, 2001.

75. Pressley, M.; Hilden, K.; Shankland, R. An Evaluation of End-Grade-3 Dynamic Indicators of Basic Early Literacy Skills (DIBELS): Speed Reading Without Comprehension, Predicting Little (Tech. Rep.); Literacy Achievement Research Center, Michigan State University: East Lansing, MI, USA, 2005.

76. Samuels, S.J. The DIBELS tests: Is speed of barking at print what we mean by fluency? Read. Res. Quart. 2007, 42, 563-566.

77. Spector, J.E. How reliable are informal reading inventories? Psychol. Sch. 2005, 42, 593-603. [CrossRef]

78. Kuhn, M.R.; Stahl, S.A. Fluency: A Review of Developmental and Remedial Practices; Center for the Improvement of Early Reading Achievement: Ann Arbor, MI, USA, 2000.

79. Rasinski, T.V.; Hoffman, T.V. Theory and research into practice: Oral reading in the school literacy curriculum. Read. Res. Quart. 2003, 38, 510-522. [CrossRef]

80. Couper-Kuhlen, E. An Introduction to English Prosody; Edward Arnold: London, UK, 1986.

81. Benjamin, R.; Schwanenflugel, P.J. Text complexity and oral reading prosody in young readers. Read. Res. Quart. 2010, 45, 388-404. [CrossRef]

82. Nevler, N.; Ash, S.; Jester, C.; Irwin, D.J.; Liberman, M.; Grossman, M. Automatic measurement of prosody in behavioral variant FTD. Neurology 2017, 89, 650-656. [CrossRef] [PubMed]

83. Ehri, L.C. Stages of development in learning to read words by sight. J. Res. Read. 1995, 18, 116-125. [CrossRef]

84. Stanovich, K.E. Matthew effects in reading: Some consequences in individual differences in the acquisition of literacy. Read. Res. Quart. 1986, 21, 360-407. [CrossRef]

85. Meyer, M.S.; Felton, R.H. Repeated reading to enhance fluency: Old approaches and new direction. Ann. Dyslexia 1999, 49, 283-306. [CrossRef]

86. Millis, K.K.; King, A. Rereading strategically: The influences of comprehension ability and a prior reading on the memory for expository text. Read. Psychol. 2001, 22, 41-65. [CrossRef]

87. Rasinski, T.V. The Fluent Reader: Oral and Silent Reading Strategies for Building Word Recognition, Fluency, and Comprehension, 2nd ed.; Scholastic: New York, NY, USA, 2010.

88. Rasinski, T.V. Why reading fluency should be hot. Read. Teach. 2012, 5, 516-522. [CrossRef]

89. Woolley, G.; Hay, I. Reading intervention: The benefits of using trained tutors. Aust. J. Lang. Lit. 2007, 30, 9-20.

90. Wolf, M.; Katzir-Cohen, T. Reading fluency and its intervention. Sci. Stud. Read. 2001, 5, 211-239.

91. Roberts, G.J.; Capin, P.; Roberts, G.; Miciak, J.; Quinn, J.M.; Vaughn, S. Examining the effects of afterschool reading interventions for upper elementary struggling readers. Rem. Spec. Ed. 2018, 39, 131-143. [CrossRef]

92. Bryk, A.S. 2014 AERA distinguished lecture: Accelerating how we learn to improve. Educ. Res. 2015, 9, 467-477. [CrossRef]

93. Kim, J.S. Making every study count: Learning from replication failure to improve intervention research. Educ. Res. 2019, 48, 599-607. [CrossRef]

94. Harris, T.L.; Hodges, R.E. The Literacy Dictionary: The Vocabulary of Reading and Writing; International Reading Association: Newark, NJ, USA, 1995.

95. Guidelines for Examining Phonics and Word Recognition Programs; Texas Reading Initiative; Texas Education Agency: Austin, TX, USA, 2002.

(C) 2020 by the authors. Licensee MDPI, Basel, Switzerland. This article is an open access article distributed under the terms and conditions of the Creative Commons Attribution (CC BY) license (http://creativecommons.org/licenses/by/4.0/). 